\title{
Bacterial Small RNA Regulators
}

Nadim Majdalani, Carin K. Vanderpool, and Susan Gottesman Laboratory of Molecular Biology, National Cancer Institute, Bethesda, MD, USA
Editor: William Reznikoff

Address correspondence to Susan Gottesman, Bldg. 37, Rm. 5132, National Cancer Institute, Bethesda, MD 20892, U.S.A.

E-mail: susang@helix.nih.gov

\begin{abstract}
Small regulatory RNAs can modify the activity of proteins and the stability and translation of mRNAs. They have now been found in a wide range of organisms, and can play previously unsuspected critical regulatory roles. The bacterial small RNAs include two major classes. The largest family (with at least 20 members in Escherichia coli K12) acts by basepairing with target mRNAs to modify mRNA translation or stability; this class of RNAs also uses an RNA chaperone protein, Hfq. DsrA is the best-studied example of this family of RNAs. It has been shown to positively regulate translation of the transcription factor RpoS by opening an inhibitory hairpin in the mRNA, and to negatively regulate translation of hns by pairing just beyond the translation initiation codon. The class of RNAs that modify activity of proteins is exemplified by CsrB and CsrC of E. coli, two RNAs that bind to and inhibit CsrA, a protein translational regulator. Homologs of CsrA and related regulatory RNAs have been implicated in the regulation of gluconeogenesis, biofilm formation, and virulence factor expression in plant and human pathogens.
\end{abstract}

KEYWORDS CsrA, CsrB, DsrA, Hfq, RpoS

\section{INTRODUCTION}

Small RNAs that do not encode polypeptides have recently seized the attention of many researchers working in both prokaryotic and eukaryotic organisms. The eukaryotic microRNAs have been found to act on translation, regulating specific genes during development, and interfering RNAs have been shown to both have naturally important roles in silencing genes and to provide an extremely useful genetic tool (McManus \& Sharp, 2003). In prokaryotes, small RNAs made from the opposite strand from their targets and therefore able to basepair extensively with their target mRNA have been described and studied as regulators of plasmid and phage functions for many years. More recent recognition of the importance of trans-acting small RNAs in regulation of bacterial gene expression, including genes involved in bacterial pathogenesis, has extended interest in these molecules, and has led to genome-wide searches for these small RNAs in E. coli, and, more recently, in other organisms as well. More than 200 have been predicted by various approaches; of these, the expression of 60 small RNAs has been confirmed by Northern blotting in E. coli K12. What do all these small RNAs do, and how do they do it? In this review, we focus on two of the most extensively studied examples, one each of two major types of non-coding regulatory RNAs. 
The first type acts by basepairing with one or more target messenger RNAs; the outcome of pairing can be activation or inhibition of translation, and/or activation or inhibition of mRNA degradation. Of the currently recognized small RNAs, at least one third and probably more act in this fashion. Many, if not all, of these also bind to and use the RNA chaperone Hfq. As an example of such a small RNA, we discuss in detail DsrA, an $E$. coli non-coding RNA with a significant role in the stimulation of translation of the stationary phase sigma factor, RpoS, as well as other cellular roles. For valuable information on the details of interactions of small RNAs with targets, the reader is recommended to consult recent reviews on the mechanism of action of plasmid-encoded small RNAs (Brantl, 2002; Gerdes et al., 1997; Wagner \& Brantl, 1998). These differ from the bacterial RNAs discussed here primarily because the plasmid RNAs are encoded on the opposite strand of their target messages, leading to extensive complementarity; the bacterial RNA genes discussed here are generally far from the genes for their targets.

The second type of non-coding RNA acts by interaction not with a message, but with a protein. CsrA is an RNA binding protein that acts as a translational regulator in E. coli; homologs play roles in virulence in Erwinia species, and Pseudomonads as well. The activity of CsrA is at least in part modulated by interactions with non-coding RNAs that titrate CsrA away from its mRNA targets. CsrB and CsrC have been identified as such regulatory RNAs in E. coli, and homologs exist in many other organisms.

\section{TRANS-ACTING BASEPAIRING RNAs}

The small RNA DsrA will be discussed as the paradigm for small RNAs (sRNAs) that basepair with their targets. DsrA is one of the first sRNAs of this class to have been identified and has been one of the most thoroughly studied. DsrA positively regulates translation of the stationary phase sigma factor RpoS and negatively regulates translation of the histone-like protein HNS. Another small RNA, RprA, also acts positively on translation of its only known target, rpoS. However, most other basepairing small RNAs act negatively. The only other example of a regulatory RNA with both positive and negative effects is RNAIII of $S$. aureus (Morfeldt et al., 1995) involved in virulence regulation; this RNA is considerably larger than DsrA, but may act similarly in terms of pairing to targets to either stimulate or inhibit translation; it also contains a short translated region. DsrA exerts a broad effect on gene expression because both of its known targets, RpoS and HNS, are themselves global regulators; as a result, the indirect effects of DsrA via effects on these global regulators are extensive. Although the biological effects of DsrA may be particularly broad, what we know about the mechanism of action of DsrA has many similarities to the many other pairing RNAs that act negatively. These RNAs all require the RNA chaperone protein Hfq for activity, and their binding to and dependence upon Hfq defines the class of regulatory RNAs represented by DsrA. The wealth of information available about DsrA is unmatched for any of the other RNAs and therefore presents a model of successes and obstacles that research on other small RNAs may face.

\section{Discovery of DsrA}

DsrA was discovered in this laboratory accidentally during studies on capsule regulation. The transcription of genes involved in synthesis of colanic acid capsule (cps genes) in E. coli requires the RcsC/YojN (RcsD)/RcsB phosphorelay, in which RcsB is a DNAbinding response regulator (Stout \& Gottesman, 1991; Takeda et al., 2001). In addition, a second DNA binding protein, the unstable protein RcsA, is needed for stimulation of transcription of the cps genes. A multicopy plasmid encoding RcsA increased capsule synthesis, as expected because RcsA is normally limiting for $c p s$ gene expression. More surprisingly, when the $\operatorname{rcs} A$ gene on the plasmid was inactivated by transposon insertion, it still stimulated cps gene expression; stimulation required an intact $r c s A$ gene in the chromosome (Brill et al., 1988). This was originally interpreted as suggesting that the plasmid was titrating a negative regulator of $\operatorname{rcs} A$ synthesis. However, subsequent experiments demonstrated that the active portion of the plasmid was not upstream of $r c s A$ but downstream. Darren Sledjeski investigated this further and found that the relevant portion of the plasmid encoded a short RNA with no obvious open reading frames. Transcription of the RNA was necessary to stimulate capsule synthesis and mutations in either the promoter of the RNA or in the Rho-independent terminator abolished function (Sledjeski \& Gottesman, 1995). The RNA was named DsrA (downstream from $\underline{R} c s \underline{A})$.

Why did DsrA overproduction from a plasmid turn up capsule expression? Experiments on the regulation 
of $\operatorname{rcs} A$ suggested that $r \operatorname{cs} A$ transcription is negatively regulated by the pleiotropic regulatory protein HNS. In bns mutants, $r c s A$ transcription is increased, which is sufficient to make cells mucoid. DsrA overproduction resulted in an HNS mutant phenotype, relieving repression of the $\operatorname{rcs} A$ promoter, as well as other HNS target promoters (Sledjeski \& Gottesman, 1995). The mechanism of the DsrA effect on HNS is discussed further below.

Although overproduction of DsrA clearly had an effect on HNS-regulated genes, it was difficult to discern an effect of deleting the chromosomal copy of $d s r A$ on capsule or other HNS targets (Sledjeski et al., 1996). However, a reporter fusion with another gene present on the original plasmid, called $d s r B$, was stimulated by a multicopy $d s r A$ plasmid, and mutations in $d s r A$ had a significant effect on the expression of the $d s r B-l a c Z$ transcriptional fusion, particularly at low temperatures (Sledjeski et al., 1996). While the function of DsrB was (and remains) unknown, the pattern of its expression was striking; transcription increased significantly when cells entered stationary phase, a sign that $d s r B$ transcription might be dependent upon the stationary phase sigma factor RpoS. Because capsule is made preferentially at low temperature, we examined the effect of DsrA at low temperatures and found that RpoS levels increased at low temperature, an increase that was fully dependent on $d s r A$ (Sledjeski et al., 1996). The increase was demonstrated to be due to increased RpoS translation. Because RpoS levels increased at temperatures of $30^{\circ} \mathrm{C}$ and below, even in exponential phase when RpoS levels are usually low, this suggested a critical role for DsrA and RpoS at low temperatures. These observations were the basis for the future studies on DsrA action that led to the models discussed below.

\section{Structure and Function}

DsrA is a non-coding 85 nucleotide-long small regulatory RNA. The gene sequence is very highly conserved (approximately 90\% conservation) among closely related Gram-negative organisms such as Salmonella, Shigella, \& Klebsiella. In fact, variations in the sequence of the Klebsiella DsrA, compared to that of E. coli, occur either in regions predicted to be part of single-stranded loops or, simultaneously, in opposing nucleotides in a predicted stem resulting in the maintenance of an intact stem structure. This is a clear indication of the importance of structures in the RNA, consistent with a

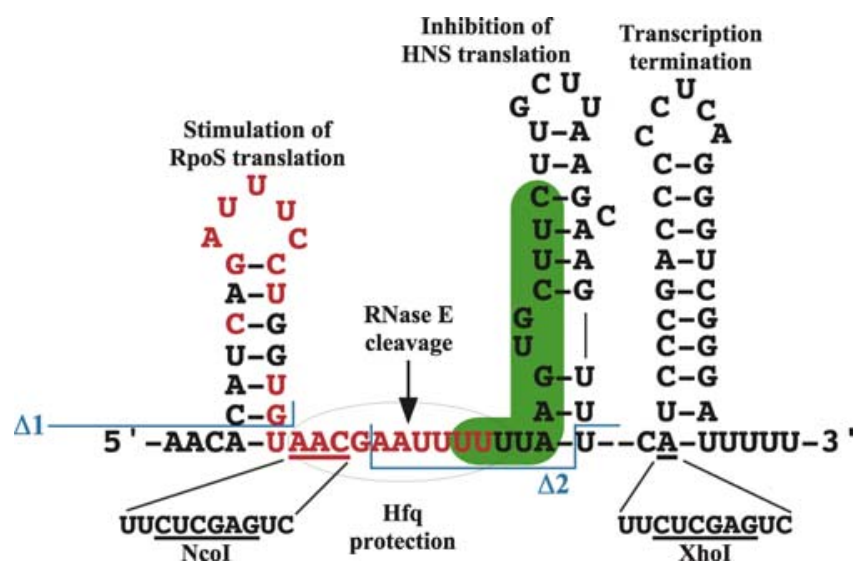

FIGURE 1 Structure of DsrA. The secondary structure of DsrA is that found in studies of in vitro nuclease sensitivity and hydroxyradical cleavage, as well as $\mathrm{Hfq}$ protection (Brescia et al., 2003; Lease \& Belfort, 2000a; Lease \& Woodson, 2004). Nucleotides in red are capable of basepairing with the rpos leader (Majdalani et al., 1998); nucleotides highlighted with green are capable of pairing with hns (Lease et al., 1998). The RNase E cleavage site is as found by (Moll et al., 2003a). The $\Delta 1, \Delta 2$, Ncol and Xhol mutations are as described in (Majdalani et al., 1998).

direct role for the RNA. RNA folding programs such as MFOLD predict a number of conformations for this RNA, but two energetically favored folds shape the RNA into a three stem-loop structure (Sledjeski \& Gottesman, 1995). While the initial stem-loop (SLI) and the terminator stem-loop (SLIII) are the same in both predictions, the middle (SLII) originally predicted was not the structure found in a nuclease footprinting analysis of in vitro synthesized RNA (Lease \& Belfort, 2000a) (Figure 1).

How does the RNA function? As mentioned above, multicopy expression of DsrA causes both increased expression of a cps-lac $Z$ fusion, via down-regulation of HNS amounts or activity, and increased translation of RpoS. Deletion analysis and point mutations in $d s r A$ demonstrated that these two activities could be separated (Majdalani et al., 1998). RNAs with deletions and rearrangements of SLI could still down-regulate HNS function, but had lost the ability to regulate RpoS; RNAs with deletion of sequences overlapping parts of SLII still retained some activity for RpoS but were unable to down-regulate HNS (Majdalani et al., 1998) (see $\Delta 1$ and $\Delta 2$, Figure 1 ).

At the time this deletion analysis was being done, another study on the role of the Hfq protein in the regulation of RpoS translation was being carried out in Tom Elliott's laboratory (Brown \& Elliott, 1997). Hfq mutants had been found to make very low levels of RpoS 
(Brown \& Elliott, 1996; Muffler et al., 1996). Brown and Elliott selected revertants of an $b f q$ mutant that expressed an RpoS-LacZ translational reporter at a higher level in the absence of Hfq and mapped in the fusion. Their mutants defined a hairpin in the sequence upstream of the rpoS coding region; a stretch of sequence more than 60 nucleotides upstream paired with the ribosome binding region, inhibiting RpoS translation (Brown and Elliott, 1997) (Figure 2A). When the hairpin was present, RpoS translation was dependent upon Hfq and environmental signals.

After closer inspection, DsrA was found to carry a stretch of nucleotides that is complementary to the up- stream region of the RpoS message. Mutations in these nucleotides abolished DsrA activity on RpoS while compensatory mutations in the rpoS leader RNA restored activity to the mutant; this provided proof that the sRNA must pair directly with its target mRNA to act in vivo (Majdalani et al., 1998). This pairing of DsrA with the $r p o S$ mRNA leads to a stimulation of translation. While the details of activation are not yet clear, the end result is. When the hairpin loop that occludes the RpoS ribosome binding site is intact, there is low translation (Brown \& Elliott, 1997) (Figure 2A). Sequences in DsrA that are complementary to the upstream part of the leader hairpin presumably lead to freeing the

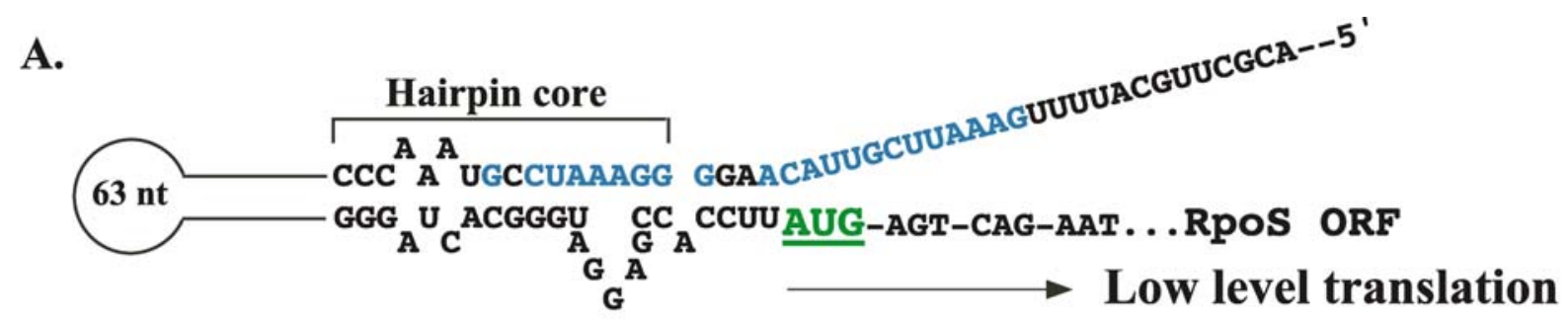

S-D

B.

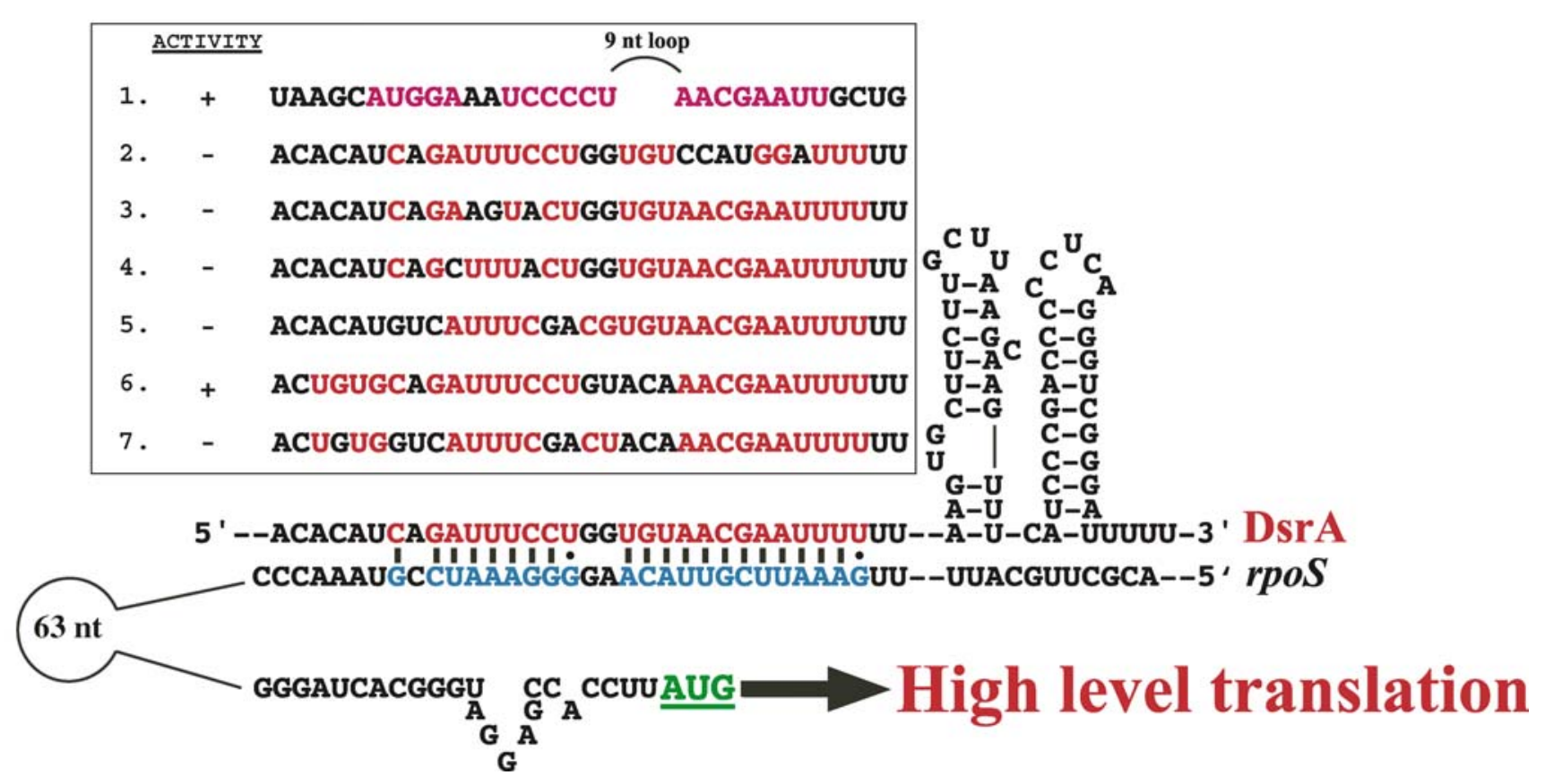

FIGURE 2 Stimulation of RpoS translation by DsrA. A. Structure of inhibitory hairpin, based on (Brown \& Elliott, 1997). Large AUG is first codon of rpoS gene. B. Pairing of DsrA, RprA, and derivatives of DsrA with rpoS upstream RNA. Pairing prediction for wild-type DsrA is from (Majdalani et al., 1998). RprA and mutant derivatives of DsrA are shown boxed, with predicted pairing in red and their activity for expression of an RpoS-LacZ fusion shown to the left. Derivatives shown: 1. Wild-type RprA (Majdalani et al., 2002). 2-7, derivatives of DsrA (see Figure 1). 2. Ncol mutation (Majdalani et al., 1998); 3. pDDS216, an unpublished variant from D. Sledjeski and N. Majdalani; 4. A-C loop mutations; the AUUUC sequence of the loop for the first stem-loop was changed to CUUUA, unpublished, N. Majdalani; 5. Inversion of top of the first stem (Majdalani et al., 1998); 6: Inversion of bottom of the first stem (Majdalani et al., 1998); 7. Inversion of all of the first stem (Majdalani et al., 1998). 
rpoS ribosome-binding site (Figure 2B). The net result is a significant increase in RpoS translation and a significant accumulation of the RpoS protein (Majdalani et al., 1998). Figure 2B summarizes some of the mutant forms of DsrA that have been tested and their effects on pairing. These results, as well as comparisons of the pairing of a second $r p o S$-stimulatory small RNA, RprA, suggest that there are at least two critical regions for pairing to the rpoS message (Majdalani et al., 1998; Majdalani et al., 2002). One is $5^{\prime}$ to the rpoS hairpin, and may provide an initial interaction site with DsrA that will be accessible even when the hairpin is present; the second region is the core of the hairpin, particularly the region that pairs to either side of the ribosome binding site loop (Figure 2A). This region is also close to the binding site for the RNA chaperone Hfq (see below), and whether pairing here occurs before or after Hfq interactions is unclear.

These results demonstrated that direct pairing of DsrA with the rpoS mRNA was necessary for DsrA action. Does DsrA also regulate HNS by pairing? In initial experiments (Sledjeski \& Gottesman, 1995), the levels of HNS protein were not found to decrease significantly upon overexpression of DsrA, suggesting a post-translational role for DsrA. However, later studies by Lease and coworkers found a two to threefold decrease in HNS protein upon DsrA overproduction, and computational analysis suggested that SLII, shown to be necessary for HNS regulation, had the potential to pair with a portion of the hns mRNA for a $13 \mathrm{nt}$ stretch just beyond the start of translation (Lease et al., 1998). Indeed, five point mutations in SLII caused DsrA to lose its anti-HNS activity; compensatory mutations in the hns mRNA restored it, confirming that this pairing is required for the inhibition of HNS translation (Lease et al., 1998). Further pairing between DsrA and the 3' end of the hns mRNA is predicted in silico, but this pairing, as well as potential pairings to several other candidate DsrA targets, have not been tested experimentally (Lease \& Belfort, 2000a). It is intriguing and thus far unexplained that a relatively moderate decrease in HNS levels has a dramatic effect on HNS activity.

\section{Role of Hfq in DsrA Function}

The demonstration that both DsrA and the Hfq protein were necessary for high levels of RpoS translation suggested that Hfq might act to help DsrA function. This has proven to be the case. Hfq was first found as an $E$. coli protein that is required for the RNA phage $\mathrm{Q} \beta$ to replicate in vitro. Hfq binds phage RNA tightly and destabilizes a secondary structure at the $3^{\prime}$ end of the phage RNA (Franze de Fernandez et al., 1968). More recently, Hfq was shown to form a multimeric ring; its structure is very similar to that of eukaryotic splicing proteins of the Sm family (Sauter et al., 2003; Schumacher et al., 2002).

The involvement of Hfq in RpoS translation and its ability to bind RNAs suggested that it might be involved in DsrA action. In addition, Hfq was found to bind another small regulatory RNA, OxyS, which negatively regulates rpoS (Zhang et al., 1998). Sledjeski and coworkers tested the in vivo role of Hfq on the activity of DsrA and it was immediately clear that Hfq was important in the DsrA-mediated regulation of both RpoS and HNS (Sledjeski et al., 2001). In the absence of Hfq, the effects of DsrA were more than 30-fold reduced on RpoS and eightfold reduced for the HNSsensitive $\operatorname{rcs} A$ promoter. Furthermore, DsrA RNA made from the chromosomal copy of the gene was unstable in an $h f q$ mutant background. However, the requirement for Hfq was not absolute. Sledjeski and coworkers detected a weak but clear DsrA-dependent, Hfqindependent activation of RpoS-LacZ when DsrA was overproduced from a multicopy plasmid. The stability of over-expressed, plasmid encoded DsrA was independent of Hfq (Sledjeski et al., 2001). The authors surmised that the abundant, plasmid-encoded DsrA was only partially folding into the correct conformation and activating RpoS-LacZ, while the rest was folding or aggregating into a nuclease-resistant but inactive conformation (Sledjeski et al., 2001). Nonetheless, these results suggested that Hfq played the role of a chaperone protein that would either help fold the small RNA into a nuclease-resistant active form and/or directly hinder the access of the nuclease by binding to DsrA.

This pattern of dependence upon Hfq for RNA stability and activity has been found with many other small regulatory RNAs (Møller et al., 2002a). About a third of the known small RNAs isolated in E. coli are bound by Hfq, and binding to Hfq can be used to identify new small RNAs (Zhang et al., 2003). In every case that has been studied, small RNAs that bind to Hfq act on mRNAs by pairing with specific mRNAs. In vitro, Hfq has been shown to bind to small RNAs and stimulate their pairing to target mRNAs, supporting a role for Hfq beyond stabilization of the small RNAs. The binding site on the small RNAs was defined as a single-stranded 
A-U rich stretch, followed by a stem (Moll et al., 2003b; Møller et al., 2002a; Zhang et al., 2002).

A number of studies have investigated whether Hfq can change RNA structure; the results suggest that it can, but does not necessarily do this in all cases. $\operatorname{sod} B$ mRNA, a target of the small RNA RyhB, is remodeled by Hfq binding to an A-U rich sequence; this remodeling frees a region that can pair with RyhB (Geissmann \& Touati, 2004). Hfq, like another RNA chaperone, StpA, could help promote splicing of a T4 phage gene, suggesting an effect on the structure of the splicing RNA, although whether this was a direct or indirect effect was not examined (Moll et al., 2003b). In in vitro experiments, the nuclease cleavage pattern of $\operatorname{omp} A$ mRNA was changed in the presence of Hfq, again suggesting the possibility that Hfq modulates RNA structures (Moll et al., 2003b). In the case of DsrA and $r p o S$, no major change in RNA structure has been detected upon Hfq binding (Brescia et al., 2003; Lease \& Woodson, 2004). The tightest binding of Hfq to the rpoS target mRNA is within the 63 nt RNA between the upstream inhibitory stem of the hairpin and the ribosome binding site (Lease \& Woodson, 2004). Binding to bns mRNA has not yet been reported. Therefore, there is thus far no evidence that Hfq helps DsrA function by changing the availability of the pairing regions.

Hfq helps stabilize DsrA in vivo, presumably by binding to it and protecting it from RNase $\mathrm{E}$ attack. The recognition site for RNase $\mathrm{E}$ is similar to that for $\mathrm{Hfq}$, a single-stranded AU-rich region. Cells defective in RNase E show higher levels of DsrA (Moll et al., 2003a); a similar sensitivity to RNase E was found for RyhB RNA in the absence of Hfq (Massé et al., 2003; Moll et al., 2003a). Competition between Hfq and RNase E has been found in other situations as well (Zhang et al., 2003). In vitro, Hfq binds DsrA in gel retardation assays (Brescia et al., 2003; Lease and Woodson, 2004; Sledjeski et al., 2001). Both nuclease protection and competition experiments using unlabeled DsrA domains to compete with the radiolabeled full-length molecule place the Hfq binding site at a single-stranded region between stems I and II of DsrA (Brescia et al., 2003; Lease \& Woodson, 2004) (Figure 1). This same region was the site of RNase E cleavage in vitro; Hfq was able to interfere with this cleavage (Moll et al., 2003a).

Is stabilization the only role for Hfq in DsrA function? This seems unlikely, given its stimulatory role in pairing of other small RNAs and their targets (Møller et al., 2002a; Zhang et al., 2002). One possible role of Hfq would be to bring together the regulatory RNA and its target message by binding both. That would imply either that a single Hfq hexamer ring can bind two different RNAs at once, or that interactions between Hfq hexamers, each binding a different RNA, can occur. The co-crystal structure of the $S$. aureus $\mathrm{Hfq}$ protein and a small AU-rich RNA oligonucleotide indicated that the RNA was wrapped in the central core of the hexamer (Schumacher et al., 2002). This may be the primary RNA binding site on Hfq. However, studies on DsrA binding to Hfq have suggested the possibility of a second binding site for RNA. Using polyU or polyA chains in a competition assay with DsrA, Brescia et al. noticed that, polyU competes with DsrA for binding, while polyA caused a supershift of the Hfq-DsrA complex, suggesting two distinct RNA binding sites (Brescia et al., 2003). Further analysis of these binding sites was performed using point mutations that altered specific amino acids that were either highly conserved or represented potential points of protein-RNA interaction in the crystal structure (Mickulecky et al., 2004). Three sets of mutations were made. A set of mutants in critical residues in the central cavity had the most dramatic effects on DsrA binding, consistent with this acting as the primary binding site for DsrA. A second set of mutants on the proximal face of the hexamer affected Hfq stability, possibly by affecting Hfq assembly. The third set of mutants, in the distal part of the Hfq structure, had effects on the binding of polyA chains, consistent with their previous proposal that Hfq indeed has two distinct binding sites. Surprisingly though, none of these mutants were defective in in vitro binding of rpoS mRNA, and only mutants in the central cavity were significantly defective for DsrA activity in vivo (Mickulecky et al., 2004). It is worth noting that Hfq has been shown to have a role in regulating polyA tailing of mRNAs, a possible small RNA-independent function for the polyA binding site (Mohanty et al., 2004); (Hajnsdorf \& Regnier, 2000).

Can a role for Hfq in helping to bring DsrA and rpoS RNA together be demonstrated directly? Thus far, in vitro studies have failed to show a strong requirement for Hfq. When both DsrA and either hns or rpoS mRNA are present together in vitro, they can anneal in the absence of Hfq, resulting in the expected changes in nuclease or hydroxy-radical sensitivity of both RNAs (Lease \& Belfort, 2000b; Lease \& Woodson, 2004); (Espinosa, submitted). The rearrangements in the rpoS 
mRNA result in the ribosome binding site becoming more accessible to single-strand nucleases, presumably reflecting accessibility to ribosomes (Lease \& Woodson, 2004), as expected from genetic experiments with DsrA (Majdalani et al., 1998). In fact, ribosome accessibility in vitro was shown to increase when an antisense oligonucleotide to the inhibitory hairpin of the rpoS 5' UTR was present (Worhunsky et al., 2003). Addition of Hfq to rpoS $5^{\prime}$ UTR and DsrA had only very mild effects on the association of these RNAs (Lease and Woodson, 2004). Annealing of rpoS 5' UTR and DsrA interfered with the primary Hfq binding site on DsrA, suggesting that Hfq may be displaced after annealing (Lease \& Woodson, 2004). Although ternary complexes with Hfq, DsrA, and rpoS leader RNA can be isolated, different studies under somewhat different experimental conditions find Hfq bound to either a site nearer the $3^{\prime}$ end of DsrA (Espinosa et al., submitted) and/or to the rpoS $5^{\prime}$ UTR (Lease \& Woodson, 2004).

Because these in vitro experiments do not provide a clear picture of why Hfq is needed for DsrA function, one possibility is that additional factors present in vivo but not in vitro increase the dependence upon Hfq. One such additional factor might be the ribosome itself. Worhunsky and coworkers found that DsrA, in the absence of detectable Hfq, binds to the $30 \mathrm{~S}$ ribosomal subunit with a binding constant similar to that of rpoS mRNA (Worhunsky et al., 2003). Binding occurred, albeit less well, with a fragment that corresponded to the first 34 nucleotides (or the $5^{\prime}$ end) of DsrA. Furthermore, DsrA binding could not be competed by tRNA or $r p o S$ mRNA, suggesting that the $30 \mathrm{~S}$ subunit may have different binding sites for mRNA and small regulatory RNAs. Such binding to the ribosome would position DsrA to act on the $r p o S$ message, freeing the ribosome binding site to immediately bind to the ribosome and begin translation, which in turn stabilizes the opening of the inhibitory hairpin (Worhunsky et al., 2003). Whether this binding to the $30 \mathrm{~S}$ subunit will prove to be physiologically relevant remains to be seen. Hfq is known to associate with ribosomal protein $S 1$ in promoting replication of the RNA phage $\mathrm{Q} \beta$ (Blumenthal \& Carmichael, 1979). Hfq and S1 have also been found to associate with RNA polymerase; in this study, an ATPase activity was found for Hfq, although no catalytic site is obvious from the sequence or crystal structures (Sukhodolets \& Garges, 2003). If Hfq either has an ATPase activity or associates with an ATPase, such an ATPase might help in remodeling the RNAs as they anneal.

\section{Additional Candidates for Modulators of DsrA}

Other proteins have also been implicated in DsrA action, although their roles are unclear. The histonelike protein HNS that preferentially binds to curved DNA (Atlung \& Ingmer, 1997) was shown to bind RNA as well. In vivo assays of DsrA stability in an hns mutant indicated that DsrA was more stable than in a wild-type strain (DsrA half-life, $18 \mathrm{~min}$ ); after an initial rapid degradation (half-life,14 $\mathrm{min}$ ), it had a halflife of $>60 \mathrm{~min}$ (Brescia et al., 2004). This effect of an hns mutant in stabilizing DsrA was even more evident in the absence of Hfq. In an $h f q$ mutant, DsrA becomes unstable, with a half-life of $2.5 \mathrm{~min}$; in the bns $h f q$ double mutant, DsrA has a half-life of $34 \mathrm{~min}$ (Brescia et al., 2004). Whether this stable DsrA is active was not determined, and because hns mutants have pleiotropic effects, it is not clear whether the effects are direct or indirect. In support of a direct interaction of HNS and DsrA, Brescia and coworkers demonstrated in vitro that HNS specifically bound DsrA (Brescia et al., 2004). In parallel to the in vivo observations, low to intermediate concentrations of HNS enhanced RNase I single-strand attack on DsrA in vitro. However, high levels of HNS protected DsrA (Brescia et al., 2004). If HNS does directly interact with DsrA, we would expect an effect in vivo on one or more of the DsrA activities. We have found that hns mutants have increased levels of RpoS, but resulting from stabilization of the protein, not increased translation (Zhou \& Gottesman, in preparation).

Another protein that seems to bind to DsrA in vitro is the histone-like protein HU. This protein binds to DNA and double-stranded RNA without any sequence specificity yet seems to recognize similar structural features in DNA and RNA (Balandina et al., 2002). Using gel retardation assays, Rouviere-Yaniv and coworkers demonstrated that HU bound full-length DsrA quite well. Using truncations of DsrA, the authors determined that $\mathrm{HU}$ was in fact binding to a region of duplex RNA preceded or followed by a region of singlestrandedness. These $3^{\prime}$ or $5^{\prime}$ overhangs are important for $\mathrm{HU}$ binding to DsrA. HU also stimulates RpoS translation (Balandina et al., 2001), leading the authors to suggest that HU may play a physiologically significant role for DsrA function in vivo (Balandina et al., 2002). 


\section{Model for DsrA Action}

Although the roles of ribosome, HNS, HU, or ribosomal proteins are not yet clear, the basic picture that we are left with is consistent with (and expands) the model derived from in vivo experiments (Majdalani et al., 1998). DsrA is synthesized at low temperatures (see below), and binds Hfq with high affinity. A site on the rpoS $5^{\prime}$ UTR is also bound by Hfq. Possibly, interactions between Hfq oligomers or binding of both RNAs to different sites on one Hfq oligomer help bring the RNAs together, enhancing pairing. Because there is no evidence that the specificity of pairing can be provided by Hfq, it seems possible that the initial pairing is stabilized by these Hfq interactions, rather than initiated by Hfq interactions. Hfq may then cycle on or off DsrA and the target mRNA, to be used again, and, in some cases, to reveal RNase E cleavage sites. While DsrA functions as a positive regulator on $r p o S$, many other small RNAs also depend upon Hfq, but lead to rapid degradation of their target messages; in those cases, displacement of Hfq would be important to allow access of the ribonuclease. It is possible that the relatively extensive base-pairing of DsrA and $r p o S$ represents one of the least Hfq-dependent small RNA targets, while for other pairs of sRNA and mRNA, Hfq stimulation of pairing will be more critical in vitro as well as in vivo. This would be consistent with the observation of some Hfq-independent DsrA activity in vivo (Sledjeski et al., 2001). Whether DsrA is normally limiting, and, if so, how its use is partitioned between $r p o S$, $h n s$, and possible other targets has not been explored at all.

\section{Regulation of DsrA Synthesis and Activity}

Perhaps the most difficult aspect of working with small RNAs is assigning them a physiological role. No small regulatory RNA has yet been found to be essential in E. coli. hfq mutants of E. coli, defective in stability and activity of many small RNAs, are also viable, albeit slowgrowing. Thus, the small RNAs may act in many cases as regulators under stress conditions or in developmental pathways, but not necessarily as critical components of vegetative growth. Consistent with a role under specific conditions, synthesis of most of these trans-acting regulatory RNAs is tightly regulated. Therefore, it is critical to find the environmental and/or physiological signals that lead to their expression to understand when the
RNA is likely to be important. Even when conditions of expression are well understood, defining the range of targets poses difficulties. The state of our information on these two issues is discussed, both generally and with respect to DsrA specifically.

DsrA was first defined by its stimulation of capsule synthesis. Because capsule synthesis occurs at low temperature, the effect of DsrA on RpoS expression at low temperature was investigated. This led to two major findings: RpoS levels increase significantly at low temperature, even in exponential phase, and this increase is completely dependent upon DsrA (Sledjeski et al., 1996). DsrA stimulation of RpoS at low, but not high, temperatures might reflect differences in synthesis, stability, and/or activity as a function of temperature. While additional effects on DsrA activity have not been ruled out, DsrA RNA accumulated at low temperature. Both synthesis and degradation were found to contribute to the higher DsrA levels (Repoila \& Gottesman, 2001).

Tests of the $d s r A$ promoter fused to the lac $Z$ reporter gene indicated that the promoter was more active at $25^{\circ} \mathrm{C}$ than at $37^{\circ} \mathrm{C}$ or $42^{\circ} \mathrm{C}$, in comparison to the lac UV5 and $\lambda \mathrm{pL}$ promoters, which were generally most active at $37^{\circ} \mathrm{C}$ and only slightly less active at $42^{\circ} \mathrm{C}$ and $25^{\circ} \mathrm{C}$. Only the region from -35 to +1 was necessary for the temperature regulation (Repoila \& Gottesman, 2001).

Promoter elements in addition to those required for temperature regulation were also identified. Promoter activity at all temperatures is enhanced by an UP element, present in promoters that contain the region from -46 to +1 . Previous work had demonstrated that the transcriptional regulator LeuO decreases RpoS synthesis when overexpressed, and acts by decreasing DsrA synthesis, in a temperature-independent manner (Klauck et al., 1997). LeuO action was localized to a region from -64 to -46 relative to the start of transcription of $d s r A$ (Repoila \& Gottesman, 2001). Whether LeuO at normal levels ever has an important role in regulating DsrA is not known, but leuO mutants have no obvious decline in $d s r A$ promoter activity.

An in-depth analysis of the $d s r A$ promoter was done using chimeric constructs between various regions of the $\operatorname{dsr} A$ and the lacUV 5 promoters. The data suggest that the $d s r A$ promoter becomes inactive at higher temperatures rather than being induced at low temperature. Analysis of the regions within the promoter necessary for temperature regulation suggest that temperature 
regulation is complex. However, the most critical element appeared to be the unusual -10 region, TAAGGT (rather than the consensus TATAAT sequence). Single nucleotide changes to bring this sequence closer to consensus (TAAAGT or TAAGAT) or to otherwise change the GG sequence (TAACCT) all led to promoters able to act at high as well as low temperatures. This -10 is absolutely dependent upon a spacer of $17 \mathrm{nt}$ for activity even at low temperature; many but not all changes in sequence of the spacer are tolerated. Finally, the distance between the -10 box and the start of transcription is also unusually short and, in conjunction with this -10 box, confers temperature dependency to the promoter. While these data do not completely eliminate the possibility of a protein factor being required for temperature regulation of the promoter, they are most consistent with a scenario where the geometry and supercoiling of the promoter DNA are responsible for low temperature activity. The regulatory information for DsrA synthesis, and therefore for RpoS synthesis, is thus thought to be intrinsic to the cellular environment that affects RNA polymerase interaction with this promoter.

\section{Targets for DsrA Action}

The initial targets of DsrA were determined as a result of the serendipitous observations on the phenotypes of overproduction of this RNA as discussed earlier. Increased capsule synthesis was traced to the antiHNS activity of DsrA, and the increase in expression of a fusion to a neighboring gene as cells entered stationary phase was traced to the positive effect of DsrA on RpoS translation (Sledjeski \& Gottesman, 1995; Sledjeski et al., 1996). Are these the only targets of DsrA, and, if not, how can one identify others? This is a general problem faced in the analysis of the many other small RNAs that act by pairing to mRNAs.

Two general approaches, each with its own problems, may define targets for a given small regulatory RNA. The first approach is to use computational means to predict potential pairing between the small RNA and other RNAs in the cell. In general, the region of pairing between the small RNA and its target can be anywhere from 7 to 20 nucleotides; whereas 15 to 20 nucleotide matches may be possible to find computationally, shorter matches are much more difficult. Programs used for such searches also do not usually recognize a $\mathrm{G}-\mathrm{U}$ pairing even though this pairing occurs in RNA.
While this problem can be addressed, some small RNAs (such as OxyS on $f b l A$ and RprA on $r p o S$, as well as DsrA on bns) have two regions of pairing with their target, separated by stretches of unpaired nucleotides on either the small RNA or the target message (Argaman \& Altuvia, 2000; Lease \& Belfort, 2000a; Majdalani et al., 2002). Nevertheless, Lease and colleagues used this approach to predict several potential targets (Lease et al., 1998), most of which have not yet been confirmed experimentally. Computational approaches have also been useful in finding targets for RyhB (Massé \& Gottesman, 2002) and Spot 42 (Møller et al., 2002b), and are likely to improve as we learn more about the behavior of these small RNAs.

The second general approach to identifying targets is to look for the outcome of small RNA expression. This can be done most globally using either microarrays or proteomic approaches. Microarrays can be useful in identifying potential direct targets if the target mRNA is expressed under the growth condition to be tested and if the small RNA leads to rapid target mRNA degradation, as is true for a number of small RNAs. For instance, RyhB expression causes a rapid decrease in target mRNA abundance by 5- to 10-fold (Massé \& Gottesman, 2002), but in other cases the changes in target mRNAs may be less or absent.

In the case of DsrA, while hns mRNA is destabilized when DsrA is expressed, the effects are not as dramatic as those for RyhB (Lease \& Belfort, 2000b). In addition, because DsrA regulates RpoS and HNS, both themselves global regulators, detecting what is a direct and what is an indirect effect of DsrA can be particularly daunting. A recent publication by Lease and colleagues reporting a role of DsrA in acid resistance illustrates the complexity of interpreting array data and distinguishing direct from indirect DsrA targets (Lease et al., 2004). The authors used microarrays to look at the profile of $E$. coli transcripts upon overproduction of DsrA; two genes whose expression was significantly elevated, $h d e A$ and $h d e B$, as well as a number of other genes less dramatically affected, such as $\operatorname{gad} A$ and $\operatorname{gad} X$, are involved in acid resistance. A $d s r A$ mutant affected in its ability to pair with the $h n s$ mRNA did not show the increase in these transcripts, consistent with a role for DsrA and not other plasmid sequences.

Finally, a $d s r A$ mutant was much more sensitive to acid treatment than a wild-type strain. Both of the major known DsrA targets have an effect on acid resistance, 
with HNS acting negatively and RpoS acting positively (see Waterman \& Small, 2003), so this result would not be unexpected. Consistent with an indirect effect, both the gad genes and $h d e$ genes are known to be regulated by RpoS and/or HNS (Arnqvist et al., 1994; Waterman \& Small, 2003). These genes are also part of a complex regulatory network (Masuda \& Church, 2003). Thus, it is quite possible that the effects of DsrA on the acid resistance genes are indirect. Nonetheless, the results do demonstrate a role for DsrA, direct and/or indirect, in acid resistance.

Given that DsrA is already known to directly regulate two genes ( $h n s$ and $r p o S$ ) in a direction consistent with increased acid resistance, does it also have other direct targets with a role in acid resistance? To answer this, it is necessary to sort out direct from indirect effects. While this is not simple, it is possible. For instance, in the case of DsrA effects on acid resistance, Lease and coworkers found that a plasmid expressing a mutant form of DsrA unable to pair with bns and shown previously, under other conditions, to have decreased stimulation of RpoS, partially complements the acid-sensitive phenotype of the $d s r A$ mutant, which led them to suggest targets other than RpoS and HNS were involved (Lease et al., 2004). Such specificity mutants have the potential to define direct versus indirect effects. No measurements of either RpoS or HNS levels were carried out in these strains to conclusively determine whether the mutant DsrA is affecting these proteins under the acid shock conditions. It is possible, for instance, that even the mutant DsrA, in combination with other environmental cues from low acid, stimulates RpoS and/or represses HNS sufficiently to complement. In a somewhat parallel case, osmotic shock induction of RpoS is dependent upon DsrA at $37^{\circ} \mathrm{C}$, although $d s r A$ mutants have very little effect on basal levels of RpoS at $37^{\circ} \mathrm{C}$, suggesting that even sub-optimal levels of this small RNA may function effectively under some conditions (Majdalani et al., 2001). A particularly useful experiment would have been to determine if the $h n s$ allele that restores pairing with the mutant $d s r A$ would restore full complementation of acid resistance (and restore induction of the acid resistance genes). If so, this would provide evidence that the acid sensitivity was a result of the anti-HNS phenotype of DsrA; if instead complementation was not restored, the role of hns could be ruled out. Fully understanding how DsrA (and other small RNAs) acts will require sorting out such direct and indirect targets.
Although arrays are particularly useful when small RNAs affect the level of their target mRNAs, other approaches can be useful because they are independent of the exact mechanism of action. Studying growth under various conditions and/or the expression of reporter fusions under conditions of over-expression or absence of the small RNA has been productive in a number of cases, although they too are not guaranteed to give direct rather than indirect targets. The identification of a phenotype allows further genetic experiments to work back to the direct targets. The inability of cells overproducing RyhB to grow on succinate (Massé \& Gottesman, 2002) and the inability of cells overproducing SgrS to grow on glucose (Vanderpool and Gottesman, 2004) were critical clues to their function, and in fact provided direct targets for these small RNAs.

\section{Physiological Roles of DsrA and Other Regulatory RNAs in Regulation of RpoS}

What does DsrA do? DsrA is expressed best at low temperatures (around $25^{\circ} \mathrm{C}$ ). Promoter studies confirmed that the promoter is active at low temperatures and decreases in activity at higher temperatures. Furthermore, the expression of RpoS is DsrA-dependent at $25^{\circ} \mathrm{C}$. All this would suggest that DsrA is more important for growth at low temperature or under other conditions that might have similar effects on the $d s r A$ promoter. From these data we can start to form an image of the physiological role of DsrA: cold temperatures are perceived as a stressful event for the bacterium and it has to respond accordingly. This predicts that among the genes activated by RpoS and/or repressed by HNS will be genes important for low temperature survival or growth. One such set of genes are those necessary for the production of trehalose, a disaccharide that seems to protect bacterial cells against cold damage. The ots $A$ and ots $B$ mRNA levels show a marked increase when cells are grown at $4^{\circ} \mathrm{C}$. This leads to an accumulation of trehalose in those cells and results in an increased viability at low temperature (Kandror et al., 2002). Trehalose synthesis is dependent on RpoS. While Kandror and coworkers did not test the DsrA dependence of this synthesis, RpoS expression at low temperature is dependent on DsrA (Sledjeski et al., 1996), so we would predict that $\operatorname{ds} A$ mutants should be defective in trehalose synthesis and therefore in cold resistance. Whether other 
RpoS-dependent genes are also needed at low temperature is not known.

The effects of DsrA on HNS and HNS-regulated genes have been observed when DsrA is overproduced. Whether this regulation is seen under physiologically relevant conditions is not yet known. Other postulated targets for DsrA (Lease et al., 1998) also remain to be explored, although preliminary data from microarrays (Lease et al., 2004) as well as other data from this lab supports a role in regulation of the $r b s$ operon (J. Wright, Y. Zhou, \& S. Gottesman, unpublished observations). The ability of a small RNA like DsrA to regulate multiple targets provides a novel mechanism of coordination of gene expression, independent of the transcriptional regulation of the individual targets. If this coordination is important, we would predict that the conditions which require DsrA-dependent RpoS translation would also require or be helped by the regulation of other DsrA targets. DsrA is not the only small RNA that regulates RpoS translation. A $d s r A$ mutant has decreased activity of an RpoS-LacZ reporter fusion, a fact that was used to screen for suppressors of the $d s r A$ null mutation. The multicopy screen yielded RprA, a second small RNA that is capable of stimulating RpoS translation by pairing to similar regions in the $r p o S 5^{\prime}$ UTR as DsrA (Figure 2B) (Majdalani et al., 2001; Majdalani et al., 2002). However, since cells do not become mucoid when overexpressing RprA, we conclude that RprA does not have the same additional targets (bns) as DsrA. RprA also differs from DsrA in when it is made. It is under the tight regulation of the $\mathrm{Rcs} C / \mathrm{Rcs} \mathrm{D} / \mathrm{RcsB}$ phosphorelay, which is activated by thus-far undefined stresses to the cell surface (Majdalani et al., 2002). Presumably, yet other RpoS targets are important for dealing with this stress. OxyS, another small RNA, negatively regulates RpoS translation, possibly by competing for Hfq (Zhang et al., 1998). Finally, yet other small RNAs may stimulate RpoS. Two other small RNAs detected in a global search in E. coli were able to stimulate expression of an RpoS-LacZ reporter and are currently under study (Wassarman et al., 2001). These small RNAs share with RprA and DsrA the ability to pair with the rpoS 5' UTR, but share little else, possibly reflecting their activities on other targets, presumably important under the specific conditions of synthesis of that small RNA. This pattern of multiple small RNAs acting on a single target is certainly not unique. In $V$. cholerae and other Vibrio species, four small RNAs are an essential part of the pathway for signaling in response to quorum sens- ing; in this case, at least part of the regulatory signals for making the small RNAs are the same, and only deletion of all four RNAs abolishes the downstream effect (Lenz et al., 2004). In $P$. aeruginosa, tandem small RNAs are part of the response to iron limitation (Wilderman et al., 2004). Nonetheless, the diversity of small RNAs regulating $r p o S$ stands as a special case.

\section{Future Directions and Unanswered Questions}

While DsrA is among the best-studied of the small RNAs, there is still much we do not understand. What is required for efficient competition between DsrA and sequences around the rpoS binding site for binding to the inhibitory stem of the rpoS message? The importance of a few pairing regions have been tested, but the contribution of others, if understood, might help in evolving more general rules for the pairing of other regulatory RNAs with their target mRNAs. One example of how a more detailed study of DsrA can be useful is a recent study aimed at perfecting RNA regulators for biotechnology uses (Isaacs et al., 2004).

Where did DsrA and related regulatory RNAs come from? Are they recently evolved regulators? DsrA itself is conserved in closely related organisms, as is RprA. It is not yet clear whether RpoS, which is quite widespread in bacteria, is regulated by comparable mechanisms in some less closely-related organisms.

\section{PROTEIN-BINDING RNAs: CsrB/CsrC/CsrA}

A class of untranslated RNA molecules that function in an Hfq-independent fashion is exemplified by the CsrB/C RNAs of the carbon storage regulatory system of E. coli. Rather than mediating their effects through base-pairing with other RNA molecules, these RNAs function by binding to the global regulatory protein CsrA and inhibiting its activity (Romeo, 1998). Other members of this family of regulatory RNAs include homologs of $\mathrm{CsrB} / \mathrm{C}$ in other organisms that also bind to and inhibit the function of CsrA-like proteins. Another regulatory RNA that modulates the function of a protein is the 6S RNA, which forms a structure that may mimic an open promoter complex that is recognized and bound by the RNA polymerase holoenzyme containing the vegetative sigma factor, $\sigma^{70}$ (Wassarman \& Storz, 2000). 


\section{Function of CsrA-Like Proteins}

Because the primary function of non-coding RNAs of the CsrB/CsrC family is to inhibit the activity of their target CsrA-like proteins, the physiological processes that are controlled by these regulatory proteins in various organisms must first be considered. A global regulator of carbon metabolism, CsrA (Carbon Storage Regulator), was first identified by transposon mutagenesis followed by screening for mutants with altered glycogen accumulation phenotypes (Romeo et al., 1993). A mutant strain with greater than 20 -fold higher levels of glycogen than wild-type was identified and the transposon insertion was mapped to a small open reading frame (ORF) at approximately 60 minutes on the E. coli chromosome, between the alaS and $\operatorname{ser} V$ genes. This ORF encoded a 61-aa polypeptide that was designated CsrA (Romeo et al., 1993). CsrA mutants display a pleiotropic phenotype, with alterations in gluconeogenesis (Sabnis et al., 1995) and glycogen accumulation, a larger cell size and altered cell surface properties (Romeo et al., 1993). CsrA mutants are non-motile (Wei et al., 2001) and show increased adherence properties and the tendency to form biofilms (Jackson et al., 2002; Romeo et al., 1993).

CsrA functions as a negative regulator of genes involved in gluconeogenesis and glycogen biosynthesis and catabolism (Liu et al., 1995; Romeo et al., 1993; Yang et al., 1996). The mechanism of negative regulation has been examined in two cases. For the $g l g C$ gene, encoding a glycogen biosynthetic enzyme, CsrA binds to two sites in the $5^{\prime}$ region of the message; one site overlaps the ribosome binding site, and the other is positioned $25 \mathrm{nt}$ upstream of the ribosome binding site within a short hairpin structure (Baker et al., 2002). Binding of CsrA to these two sites within the $g \lg C$ leader sequence blocks ribosome binding and translation of the message, and also results in destabilization of the $g l g C$ mRNA (Liu et al., 1995). Another target of CsrA negative regulation is the cst $A$ mRNA, which encodes a peptide transporter induced by carbon starvation (Schultz \& Matin, 1991). CsrA has been found to bind to the cst $A$ mRNA leader region and block ribosome access (Dubey et al., 2003). The effect on cstA mRNA stability as a result of CsrA binding and translational inhibition has not been examined. Cells lacking CsrA express higher levels of activity of several enzymes required for gluconeogenesis, including fructose-1-6-bisphosphatase, phosphoenolpyruvate synthase, and phosphoglucomutase (Sabnis et al., 1995), suggesting that these are additional targets of CsrA negative regulation. However, it is currently unclear whether this effect is mediated directly through CsrA binding to the mRNAs encoding these enzymes.

CsrA-dependent positive regulation of target genes has been reported, but the mechanism has been examined in only one case. The finding that $\operatorname{csr} A \mathrm{mu}-$ tants were non-motile (Wei et al., 2001) suggested that CsrA may be required for positive regulation of genes involved in flagellar biosynthesis. Expression of the $f l h D C$ genes, encoding the master regulators of flagellar biosynthesis, was found to be directly stimulated by CsrA, using FlhDC'-'LacZ translational fusions, and coupled in vitro transcription-translation of $f l b D C$ in extracts with or without CsrA. Analyses of the $f l b D C$ mRNA showed that this message was three- to fourfold more abundant in wild-type cells compared with $\operatorname{csr} A$ mutant cells, consistent with the observation of an approximately three-fold decreased $f l b D C$ mRNA halflife in the mutant cells. RNA gel mobility shift assays showed specific binding of CsrA to the $f l b D C 5^{\prime}$-leader sequence, suggesting that stabilization of $f l b D C$ mRNA is indeed a direct consequence of CsrA recognition and binding of this transcript (Wei et al., 2001). Other positively regulated targets included some glycolytic enzymes, including phosphoenolpyruvate carboxykinase, glucose-6-phosphate isomerase, and triose phosphate isomerase; their activity was shown to be stimulated by CsrA but the mechanism is unknown (Romeo et al., 1993; Sabnis et al., 1995).

Similar regulators have been identified and studied in a number of other gram-negative bacteria. The enterobacterium Erwinia carotovora subspecies carotovora $(E c c)$ is a plant pathogen that causes soft-rot disease in a variety of plants. Various extracellular enzymes produced by the bacterium in response to quorumsensing signals ( $\mathrm{N}$-acyl homoserine lactones, AHLs) are required for the pathogenesis of the organism (Collmer $\&$ Keen, 1986). In a genetic screen for transposon mutants that produced these virulence factors in a celldensity independent fashion, the $\operatorname{rsm} A$ (Repressor of Secondary Metabolites) gene was identified. Cells lacking $\mathrm{RsmA}$ produced the extracellular enzymes required for Ecc pathogenesis in the absence of AHLs and produced a more severe soft-rot disease than wild-type rsm $A^{+}$bacteria (Chatterjee et al., 1995). Sequencing of the $\operatorname{rsm} A$ locus revealed that the 61 aa Ecc RsmA protein was $95 \%$ identical to the E. coli CsrA protein. In fact, 
heterologous complementation studies showed that the Ecc $\operatorname{rsm} A$ gene could complement an E. coli $\operatorname{csr} A$ mutant strain to restore glycogen accumulation to wildtype levels (Cui et al., 1995), indicating that RsmA and CsrA were functionally interchangeable. While the level of homology between E. coli CsrA and Ecc RsmA suggests that the mechanism of RsmA action on its target genes may be similar to that of CsrA, this has not been conclusively demonstrated. However, in one study, it was found that mRNA levels of an $E c c$ gene $b r p N_{E c c}$, which encodes a molecule that elicits a hypersensitive reaction in plant hosts, were greatly increased in an rsm $A$ mutant compared with the wild-type strain (Cui et al., 1996), consistent with the idea that RsmA negatively modulates the expression or stability of its target transcripts.

In Pseudomonas aeruginosa, an opportunistic human pathogen, RsmA was identified by homology to CsrA of E. coli and RsmA of Ecc (Pessi et al., 2001). The P. aeruginosa $\mathrm{RsmA}$ is a 61 aa protein sharing $92 \%$ identity with CsrA of E. coli, and can functionally complement an $E$. coli $\operatorname{csr} A$ mutant for the glycogen accumulation phenotype (Pessi et al., 2001). Similar to the role of RsmA in $E c c$, in P. aeruginosa, RsmA overexpression reduced the production of secreted virulence factors by more than $80 \%$, suggesting that RsmA may negatively regulate the genes encoding these factors. Further, the levels of AHLs in P. aeruginosa, known to be involved in positive control of virulence factors, were also reduced by RsmA overexpression. However, addition of exogenous AHLs to RsmA-overexpressing cells failed to restore production of these factors, suggesting that RsmA also has a direct role in regulating virulence gene expression (Pessi et al., 2001). In addition to these negative regulatory effects, RsmA has also been reported to positively regulate expression of rhamnolipids, which are required for swarming motility in $P$. aeruginosa (Heurlier et al., 2004). In Pseudomonas fluorescens CHA0, a plant symbiont, the RsmA protein has also been shown to influence production of secreted products and secondary metabolites (Blumer et al., 1999; Heeb et al., 2002). To date, none of these regulatory effects have been demonstrated to be the result of a direct interaction between Pseudomonas RsmA and mRNA transcripts. Recently, a second CsrA-like protein that works in the same pathways, RsmE, was also identified in P. fluorescens (Reimmann et al., 2004).

CsrA/RsmA functional homologs have been identified in a variety of other bacterial species (White et al.,
1996), and their global regulatory roles are just beginning to be elucidated. In Salmonella enterica serovar Typhimurium, CsrA is involved in regulation of genes required for invasion of eukaryotic cells (Altier et al., 2000). In the intracellular pathogen Legionella pneumophila, CsrA was found to repress traits involved in transmission, including motility and pigment production (Fettes et al., 2001; Molofsky \& Swanson, 2003). Other preliminary reports have identified and examined the physiological role of CsrA homologs in the bacteria Helicobacter pylori (Barnard et al., 2004), Proteus mirabilis (Liaw et al., 2003), and Serratia marsescens (Ang et al., 2001). Furthermore, when the E. coli CsrA protein is used as a query in a BLAST search at the NCBI, CsrA homologs are found in a broad range of bacterial species, including but not limited to Yersinia species, Photorhabdus luminescens, Shewanella oneidensis, Azotobacter vinelandii, Vibrio species, Xanthomonas campestris, Coxiella burnetii, and Haemophilus influenzae.

\section{Regulatory RNAs That Act on CsrA Family Proteins}

Non-coding RNA molecules have been found to modulate the activity of CsrA/RsmA proteins; they appear to work by binding to the CsrA proteins, in competition with the mRNA targets that the proteins work on. We consider the available data on the mode of action in the next section. CsrB was the first RNA of this class to be described, and it was discovered fortuitously by virtue of its strong interaction with purified CsrA protein (Liu et al., 1997). Other CsrB-like RNAs were found as multicopy suppressors of CsrA overproduction phenotypes; these include CsrC of E. coli (Weilbacher et al., 2002), PrrB of P. fluorescens F113 (Aarons et al., 2000), and $\mathrm{RsmB}$ (formerly designated aepH locus) of $E c c$ (Liu et al., 1998; Murata et al., 1994). Two regulatory RNAs that control RsmA activity in P. fluorescens CHA0 were designated RsmZ and RsmY. RsmZ was identified by a direct search for RNAs bound to the P. fluorescens RsmA protein (Heeb et al., 2002). RsmY was identified computationally (Valverde et al., 2003) in P. fluorescens and $P$. aeruginosa by searching for a locus homologous to one from Pseudomonas syringae (Rowley et al., 1993) that had characteristics similar to other CsrB-like riboregulators. Likewise, RsmZ of $P$. aeruginosa was identified based on its conserved sequence and genomic location compared with PrrB of P. fluorescens (Heurlier et al., 2004). For the purposes of this review, the properties of these RNA 
molecules will be considered as a group, with special emphasis on details that have been described for the most well characterized members of this class of RNAs, CsrB and $\mathrm{CsrC}$ of E. coli.

The CsrB RNA was discovered during the purification and in vitro characterization of CsrA (Liu et al., 1997). The purified CsrA protein was associated in a ribonucleoprotein complex with an RNA species of $\sim 350 \mathrm{nt}$ (Figure 3A). The complex was found to contain 18 molecules of CsrA per RNA molecule, with a total molecular mass of approximately $256 \mathrm{kDa}$. In vitro, CsrA-CsrB complexes were competent to inhibit expression of the CsrA target mRNA glgC, but RNA-free CsrA was more inhibitory than CsrA-CsrB complexes

A.

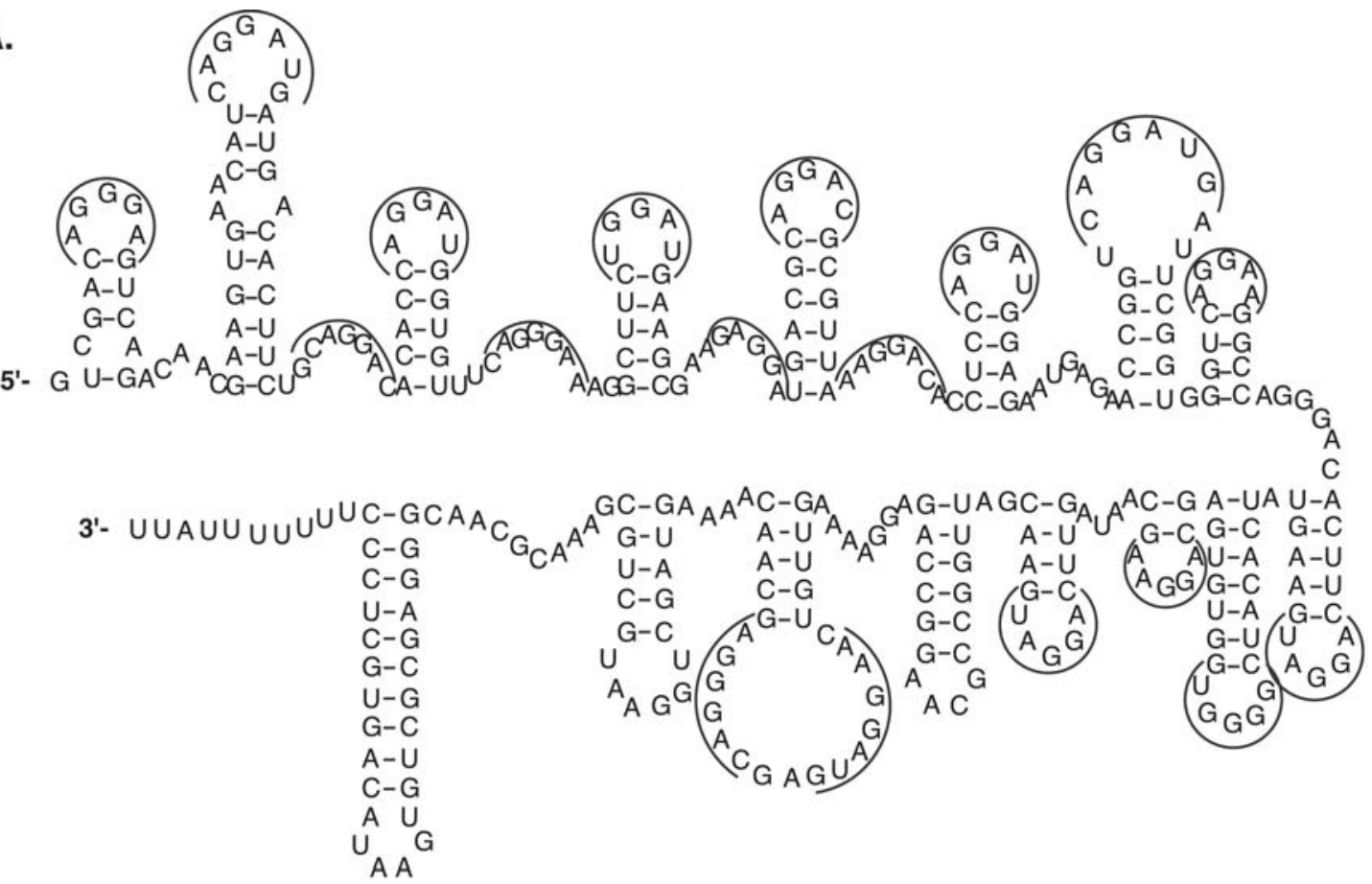

B.

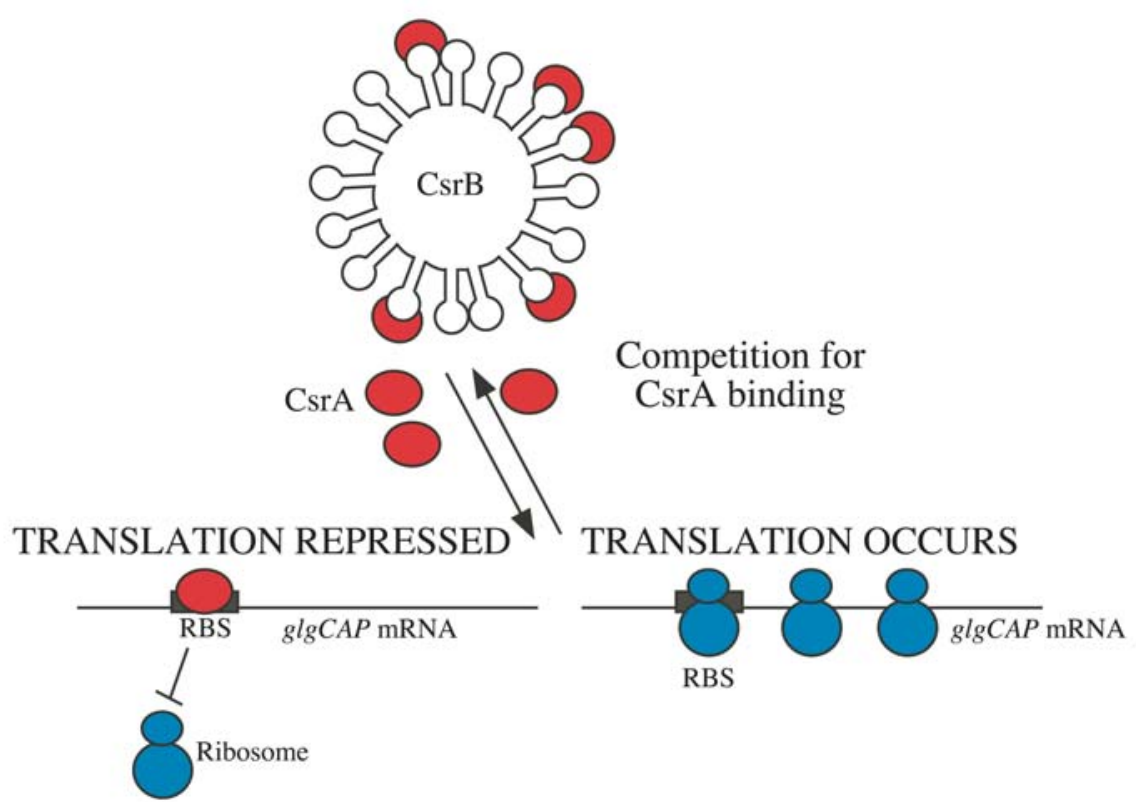

FIGURE 3 Model of CsrB and its action to inhibit CsrA. A. Structure of CsrB. The sequence of CsrB, with the regions containing CsrA binding sequences shown with arcs. The final stem-loop is a rho-independent terminator. $B$. Model of CsrB action (not to scale). CsrA protein is shown as a red oval, the ribosome in blue, and the ribosome binding site as a rectangle. CsrA can bind either to the mRNA, occluding the ribosome entry site, or to CsrB. Higher levels of CsrB are likely to compete successfully for CsrA binding, releasing the inhibition of messenger translation ( $g l g C A P$ in the figure). 
(Liu et al., 1997). The gene encoding CsrB was identified by cDNA synthesis, cloning and sequencing. The $c s r B$ gene is located at 64 minutes on the E. coli chromosome between the syd and $y q c C$ genes, more than $100 \mathrm{~kb}$ away from $\operatorname{csr} A$, and encodes a 366-nt molecule with no significant open reading frames. Within the $c s r B$ coding region, eighteen copies of a repetitive sequence (consensus 5'-CAGGA $\{\mathrm{U} / \mathrm{C} / \mathrm{A}\} \mathrm{G}-3^{\prime}$ ) were observed, and when the structure of the CsrB molecule was predicted, the majority of these consensus sequences occurred in single stranded regions of the molecule, predominantly in the loops of predicted stem-loop structures. This finding, combined with the 18:1 CsrA:CsrB stoichiometry, led to the hypothesis that these repeated sequences constituted the CsrA binding site (Liu et al., 1997). Consistent with this idea, similar sequences in the $\operatorname{glg} C$ leader region were shown to be required for CsrA binding and translational repression (Baker et al., 2002).

A second RNA molecule involved in the regulation of CsrA activity was identified in a genetic screen for plasmids carrying genomic regions that increased glycogen accumulation in E. coli cells (Weilbacher et al., 2002). This RNA was called CsrC. The $\operatorname{csr} C$ gene encodes a $245 \mathrm{nt}$ RNA molecule and is located between the yih $A$ and $y i b I$ genes, far from either $\operatorname{csr} A$ or $\operatorname{csr} B$. Putative $\sigma^{70}$ like promoter elements and a Rho-independent transcriptional terminator were identified upstream and at the $3^{\prime}$-end of the $\operatorname{csr} C$ coding sequence, respectively. Like CsrB, the CsrC molecule contained repetitive sequences similar to those found in $\mathrm{CsrB}$ and predicted to constitute CsrA binding sites; however, in CsrC there were only nine repeats (instead of 18 as in $\mathrm{CsrB}$ ).

Although CsrA-like proteins are readily identified in many sequenced bacterial genomes using BLAST analysis, it has not been possible in many cases to identify $\mathrm{CsrB} / \mathrm{C}$ homologs by nucleotide sequence similarity. While it is possible that CsrA-like proteins in some organisms are regulated by mechanisms not mediated by non-coding RNAs, the more likely explanation is that the nucleotide sequence of these RNAs has not been conserved in evolution while the structure and function has. Several examples supporting this theory of conserved structure and mechanism for CsrB/C-like RNAs will be briefly described.

The Ecc RsmB RNA exists as two different species in the cell: a long $479 \mathrm{nt}$ full-length species (RsmB) and a $258 \mathrm{nt}$ species ('RsmB) that comprises the $3^{\prime}$ end of fulllength RsmB (Liu et al., 1998). 'RsmB is processed from full-length RsmB and is the more abundant species in vivo. This $3^{\prime}$ RsmB region was both necessary and sufficient to antagonize RsmA activity in Ecc and CsrA activity in E. coli (Liu et al., 1998). The overall level of nucleotide sequence similarity between RsmB and CsrB is low. However, RsmB does contain repeated sequences similar to those proposed to be involved in CsrA binding in E. coli, which may account for the ability of $E c c$ rsmB to complement E. coli csrB mutants. The role of $\mathrm{RsmB}$ processing in the regulation of RsmB activity in $E c c$ has not yet been examined.

The regulatory RNA molecule PrrB, in P. fluorescens F113, bears little sequence similarity to $E$. coli $\mathrm{CsrB}$ and CsrC. The PrrB molecule is only 133 nt long (Aarons et al., 2000), considerably smaller than the CsrB, CsrC, and $E c c$ RsmB molecules. However, secondary structure predictions showed a PrB structure with eight stemloops containing nine imperfect 5'-AGGA-3' repeats predominantly in the loops or other unpaired regions of the RNA, a configuration strikingly similar to that predicted for CsrB and CsrC.

The RsmZ RNA of $P$. fluorescens CHA0 was initially found using an experiment to identify RNAs bound to the P. fluorescens CHA0 RsmA protein (Heeb et al., 2002). Cloning and sequencing of the $r s m Z$ gene revealed that it shared $72 \%$ identity with $\operatorname{PrrB}$ of P. fluorescens F113 and was also localized to the same genomic region, between the $r p o S$ and $f d x A$ genes. Like PrrB, RsmZ of $P$. fluorescens CHA0 showed very little sequence similarity to E. coli $\mathrm{CsrB}$ or $E c c$ RsmB; however, the conserved stem-loop structure with repetitive sequence motifs was present. The RsmZ RNA exists in vivo as a full-length $127 \mathrm{nt}$ species and a $92 \mathrm{nt}$ species lacking the $3^{\prime}$ terminator region. The contribution of each of these molecules to RsmZ function has not been described. The second RsmA activity-modulating RNA in P. fluorescens CHA0 is RsmY, which was identified by computational prediction (Valverde et al., 2003). In $P$. fluorescens CHA0, the rsm $Y$ gene was found to be located in an intergenic region between a putative acyl-CoA dehydrogenase (homologous to PA0508 in P. aeruginosa) and a putative LysR-like transcriptional regulator (homologous to PA0528 in P. aeruginosa). The rsm $Y$ gene is also found in this genomic location in other sequenced Pseudomonas species. The RsmY RNA is $118 \mathrm{nt}$ and like RsmZ, is predicted to contain stem-loop secondary structures with characteristic repeat sequences in the loops and unpaired regions of the molecule (Valverde et al., 2003). 
As in the other Pseudomonas species, the P. aeruginosa $r s m Z$ gene was identified in the $r p o S-f d x A$ intergenic region (Heurlier et al., 2004). While RsmZ sequence conservation among pseudomonads is only $\sim 45 \%$, the $P$. aeruginosa RsmZ also shows the conserved predicted structure and presence of repeated sequences in unpaired regions of the RNA.

\section{Mechanism of CsrB/CsrC Action}

Several lines of evidence point to a CsrA binding and titration mechanism for $\mathrm{CsrB} / \mathrm{CsrC}$ activity (Figure 3B). First, as described above, CsrB/C-like RNAs have been identified by virtue of their binding to CsrA homologs (Heeb et al., 2002; Liu et al., 1997). Romeo and coworkers found that each CsrB RNA was capable of binding 18 CsrA molecules (Liu et al., 1997), consistent with the idea that CsrB has the capacity to titrate CsrA away from mRNA targets. In vivo overexpression and mutant studies support this picture. Overexpression of $\mathrm{CsrB} / \mathrm{C}$-like RNAs result in phenotypes resembling those of $c s r A$ mutants. For example, overexpression of $\mathrm{CsrC}$ in $E$. coli causes a substantial increase in glycogen levels, similar to what is observed in csr $A$ mutants (Weilbacher et al., 2002). Overexpression of RsmZ in P. aeruginosa caused a loss of swarming ability, decreased lipase production, and increased pyocyanin production, all phenotypes of $\operatorname{rsm} A \mathrm{mu}-$ tant cells (Heurlier et al., 2004). Conversely, deletion of CsrB/C-like RNAs usually results in CsrA "hyperactive" phenotypes, although in at least two cases this is only seen when both $\mathrm{CsrB} / \mathrm{CsrC}$ genes are deleted, suggesting functional redundancy (Valverde et al., 2003; Weilbacher et al., 2002). Finally, in vitro experiments examining translation of a CsrA target mRNA, $g l g C$, found that RNA-free CsrA preparations were more inhibitory to $g \lg C$ translation than CsrA-CsrB complexes (Liu et al., 1997), suggesting that CsrB competes with mRNAs for CsrA binding. Whether some messages are more susceptible to competition than others is not yet clear.

\section{Regulation of csrB/csrC Expression}

Because the proposed function of $\mathrm{CsrB} / \mathrm{C}$ involves antagonism of CsrA activity, the ratio of $\mathrm{CsrA}: \mathrm{CsrB} / \mathrm{C}$ is likely to be crucial to the regulation of CsrA activity. Thus, it follows that regulation of $\mathrm{CsrB} / \mathrm{C}$ synthesis and/or stability could be important regulatory checkpoints that dictate the expression of CsrA-regulated genes. Primer extension analysis to define the $5^{\prime}$ end of the CsrB RNA showed that the promoter of the $c s r B$ gene shows significant similarity to $\sigma^{70}$-like promoters (Gudapaty et al., 2001). Northern blot analysis of CsrB levels throughout the growth curve showed that CsrB RNA levels rise modestly (three-fold) as wild-type cells enter stationary phase. Strikingly, in $\operatorname{csr} A$ mutant strains, CsrB levels were diminished approximately tenfold compared to the wild-type strain. The decrease in steady-state CsrB levels in csr $A$ mutants was not due to altered stability of $\mathrm{CsrB}$ in the absence of CsrA protein; the chemical half-life of $\mathrm{CsrB}$ was equivalent in wild-type and $\operatorname{csr} A$ null strains (Gudapaty et al., 2001). In vivo and in vitro studies provided evidence that CsrA indirectly affects transcription of $c s r B$, possibly through post-transcriptional regulation of a transcription factor required for full $c s r B$ expression; the result is a negative autoregulatory loop in which uncomplexed CsrA increases the level of its negative regulatory RNA.

In Pseudomonas species and Ecc, a two-component signal transduction system, GacA/GacS, had been implicated in regulation of RsmA targets (Blumer et al., 1999) or directly shown to regulate expression of $r s m B$ (Aarons et al., 2000; Cui et al., 2001; Heeb et al., 2002). A homologous two-component system, composed of the BarA sensor kinase and UvrY response regulator, was likewise found to control expression of $c s r B$ in $E$. coli (Suzuki et al., 2002). In a $\operatorname{csr} A$ mutant strain with decreased $c s r B$ expression, $c s r B$ transcription could be restored with either $c s r A$ or $u v r Y$ in trans. In a $u v r Y$ mutant, on the other hand, $c s r B$ expression was nearly eliminated and could only be complemented by $u v r Y$ in trans. In vitro, UvrY was also found to activate $c s r B$ transcription (Suzuki et al., 2002), strongly suggesting a direct role for $\mathrm{UvrY}$ in regulation of $\operatorname{csr} B$.

Like CsrB, CsrC levels were also diminished in csr $A$ and $u v r Y$ mutant strains (Weilbacher et al., 2002), and as with $c s r B$ expression, the effect of CsrA appeared to be indirect, while UvrY directly stimulated $\operatorname{csr} C$ transcription in vitro. Interestingly, mutation of either $\operatorname{csr} B$ or $\operatorname{csr} C$ resulted in an apparent compensatory increase in expression of the remaining gene (Weilbacher et al., 2002), though the mechanism of this compensation was not determined.

Although these studies have genetically defined factors involved in regulation of $c s r B / c s r C$ expression, the signal causing BarA/UvrY-dependent transcriptional activation of these genes remains unknown. Likewise, the role of $\mathrm{Csr} A$ in influencing $c s r B / C$ transcription 
has not been investigated; a reasonable model may be that CsrA positively regulates translation of some as yet unidentified gene involved in this regulatory circuit.

As mentioned above, the involvement of BarA/UvrY in the regulation of $c s r B / C$ of $E$. coli was first hypothesized based on comparison to regulatory studies in $P$. fluorescens and $E c c$ showing the role of GacAS in regulation of $r s m B(E c c)$ and $r s m Z$ (P. fluorescens CHA0). In these organisms, gac $A S$ mutants were found to have phenotypes similar to those of RsmA-overexpressing strains; that is, expression of RsmA-target genes was significantly lower than in wild-type strains (Blumer et al., 1999; Cui et al., 2001; Heeb et al., 2002; Hyytiainen et al., 2001). In P. fluorescens CHA0, the GacAS system apparently senses and responds to a non-AHL quorum sensing signal (Heeb et al., 2002), the chemical nature of which remain unknown. Thus, expression of the genes rsm $Y$ and $r s m Z$, the two RsmA-modulating RNAs in $P$. fluorescens CHA0, increases as a function of cell density, i.e., when the signal accumulates in the medium (Heeb et al., 2002; Valverde et al., 2003). Overexpression of RsmY or RsmZ suppressed the gac AS mutation and restored expression of negatively regulated RsmA targets (Heeb et al., 2002; Valverde et al., 2003). In studies examining the role of RsmY 5'-GGA-3' motifs, which were predicted to constitute the core of the RsmA binding site, it was observed that mutant RsmY molecules that bound poorly to RsmA in vitro had a significantly reduced half-life in vivo (Valverde et al., 2004), suggesting that in P. fluorescens CHA0, RsmA binding to RsmY stabilizes this RNA molecule.

In $E c c$, the regulation of the $r s m B$ gene may be more complicated than in P.fluorescens and E. coli. In addition to positive regulation of $E c c r s m B$ by GacAS, at least two other factors seem to influence transcription of $r s m B$ in this organism. A transcriptional repressor $\mathrm{KdgR}_{E c c}$ was shown to repress expression of $r s m B$ (Liu et al., 1999), while a 130 aa protein designated $\mathrm{RsmC}$ had a positive effect on $r s m B$ transcription (Cui et al., 1999). The signals to which these regulators are responding in order to modulate $r s m B$ expression have not been elucidated.

\section{Unanswered Questions and Issues}

The family of CsrA-related proteins is widespread in bacteria and regulate a variety of important functions. It seems likely that where CsrA-family proteins are found, the regulatory RNAs will be as well. What remains least clear about these regulators are the conditions under which the regulatory RNAs successfully compete for CsrA. Once a regulatory RNA has bound CsrA, is there a mechanism for dissociation and/or degradation of either RNA or protein? Is this a regulated process?

What is the advantage of redundancy in the regulatory RNAs? By comparison to what we know about pairing RNAs, such redundancy implies possible differences in both regulation of each regulatory RNA and in outcome of having a certain level of one or other RNA. This would suggest the possibility of an as yet unidentified environmental condition that enhances the expression of one regulatory RNA more than the other, coupled with a hierarchy in their ability to compete for CsrA relative to specific targets. The complexity of the possible regulatory networks is even greater when redundant regulatory proteins also exist as they do for $P$. fluorescens (Reimmann et al., 2005). Future work should clarify some of these issues.

\section{Finding Small RNAs/Targets: Past and Future Prospects}

DsrA, the small pairing RNA discussed above, was found accidentally during studies of a plasmid that carried the $d s r A$ gene. CsrB was found as an RNA that bound to the CsrA protein. A number of other small RNAs were either found by accident or during early searches for highly stable RNA species. More recently, some of the characteristics of these small RNAs have been used in global searches for yet others. The properties that have been useful in such searches are discussed below:

1) Non-coding RNAs are frequently conserved between related species, and their genes are found in intergenic regions. A number of searches have used conservation within the intergenic regions as a primary criterion (Rivas et al., 2001; Wassarman et al., 2001). A computer program, QRNA, has been developed to identify new small RNAs; the search parameters incorporate conservation in secondary structure elements (stems) typical of these small RNAs (Rivas et al., 2001). Conservation of small RNAs is sometimes even better than that for the flanking genes, although in some cases, conserved RNAs are found in different contexts in different organisms as well (Hershberg et al., 2003). It is worth noting, however, that conservation rarely extends far beyond genomes with reasonably high overall identity, at 
least at the level of small RNA sequence. Thus, E. coli small RNA homologs are directly identifiable by homology search in Salmonella, Klebsiella, Vibrio and Yersinia, but not in Pseudomonas. Therefore, finding the functionally similar small RNAs in more distant species requires other approaches.

2) These short RNAs frequently end in rhoindependent terminators; the presence of such a structure, in combination with an appropriate promoter (see below) or high conservation (characteristic 1) has successfully been used to identify small RNAs in organisms other than E. coli (Lenz et al., 2004; Wilderman et al., 2004).

3) The transcription of these small RNAs is highly regulated, frequently from recognizable promoters. In both Pseudomonas aeruginosa and Vibrio cholerae, searches for promoters of a specific type in combination with other characteristics allowed identification of appropriately regulated small RNAs (Lenz et al., 2004; Wilderman et al., 2004).

4) A subset of RNAs, such as DsrA, bind and use the RNA chaperone, Hfq. Immunoprecipitation of Hfq with bound RNAs, followed by characterization of the RNAs on arrays, has been used to identify and characterize E. coli regulatory RNAs of this family (Zhang et al., 2003). Since Hfq is well-conserved in other organisms (Sun et al., 2002), this approach should work in them as well, as should parallel approaches with other RNA binding proteins.

5) Small RNAs are small. Direct cloning of RNAs shorter than the bulk of tRNAs and mRNAs has been used in both prokaryotes and eukaryotes to identify novel non-coding RNAs (He \& Hannon, 2004; Vogel et al., 2003; Kawano et al., 2005).

Now that small RNAs have been recognized in many organisms and methods for finding them have been developed, it seems likely that understanding regulation in the future will mean not only appreciating the importance and mechanism of transcriptional regulators, but will also require understanding what small RNAs are doing to expand, upstage and modulate the efforts of the transcriptional regulatory proteins.

\section{ACKNOWLEDGEMENTS}

We thank R. Lease, D. Sledjeski, A. Feig, D. Haas, and G. Storz for sharing their results and manuscripts before publication. We thank G. Storz, T. Romeo, P. Babitzke, D. Haas, R. Lease and C. McCullen for comments on this manuscript.

\section{REFERENCES}

Aarons, S., Abbas, A., Adams, C., Fenton, A., and O'Gara, F. 2000 A regulatory RNA (PrrB RNA) modulates expression of secondary metabolite genes in Pseudomonas fluorescens F1 13. J Bacterio/ 182: 3913.

Altier, C., Suyemoto, M., and Lawhon, S.D. 2000. Regulation of Salmonella enterica serovar typhimurium invasion genes by CsrA. Infect Immun 68:6790.

Ang, S., Horng, Y.T., Shu, J.C., Soo, P.C., Liu, J.H., Yi, W.C., Lai, H.C., Luh, K.T., Ho, S.W., and Swift, S. 2001. The role of RsmA in the regulation of swarming motility in Serratia marcescens. J Biomed Sci 8:160.

Argaman, L. and Altuvia, S. 2000. fh/A repression by OxyS RNA: Kissing complex formation at two sites results in a stable antisense-target RNA complex. J Molec Biol 300:1101.

Arnqvist, A., Olsen, A., and Normark, S. 1994. $\omega^{\text {S}}$-dependent growthphase induction of the csgBA promoter in Escherichia coli can be achieved in vivo by $\sigma^{70}$ in the absence of the nucleoid-associated protein H-NS. Molec Microbiol 13:1021.

Atlung, T. and Ingmer, H. 1997. H-NS: a modulator of environmentally regulated gene expression. Mol Microbiol 24:7.

Baker, C.S., Morozov, I., Suzuki, K., Romeo, T., and Babitzke, P. 2002. CsrA regulates glycogen biosynthesis by preventing translation of glgC in Escherichia coli. Mol Microbiol 44:1599.

Balandina, A., Claret, L., Hengge-Aronis, R., and Rouviere-Yaniv, J. 2001. The Escherichia coli histone-like protein $\mathrm{HU}$ regulates rpoS translation. Molec Microbiol 39:1069.

Balandina, A., Kamashev, D., and Rouviere-Yaniv, J. 2001. The bacterial histone-like protein $\mathrm{HU}$ specifically recognizes similar structures in all nucleics acids, DNA, RNA, and their hybrids. J Biol Chem 277: 27622

Barnard, F.M., Loughlin, M.F., Fainberg, H.P., Messenger, M.P., Ussery, D.W., Williams, P., and Jenks, P.J. 2004. Global regulation of virulence and the stress response by CsrA in the highly adapted human gastric pathogen Helicobacter pylori. Mol Microbiol 51:15.

Blumenthal, T. and Carmichael, G.G. 1979. RNA replication: function and structure of QB-replicase. Annu Rev Biochem 48:525.

Blumer, C., Heeb, S., Pessi, G., and Haas, D. 1999. Global GacA-steered control of cyanide and exoprotease production in Pseudomonas fluorescens involves specific ribosome binding sites. Proc Natl Acad Sci USA 96:14073.

Brantl, S. 2002. Antisense-RNA regulation and RNA interference. Biochim Biophys Acta 1575:15.

Brescia, C.C., Mikulecky, P.J., Feig, A.L., and Sledjeski, D.D. 2003. Identification of the Hfq-binding site on DsrA RNA:Hfq binds without altering DsrA secondary structure. RNA 9:33.

Brescia, C.C., Kaw, M.K., and Sledjeski, D.D. 2004. The DNA binding protein $\mathrm{H}-\mathrm{NS}$ binds to and alters the stability of RNA in vitro and in vivo. J Molec Biol 339:505.

Brill, J.A., Quinlan-Walshe, C., and Gottesman, S. 1988. Fine-structure mapping and identification of two regulators of capsule synthesis in Escherichia coli K-12. J Bacteriol 170:2599.

Brown, L. and Elliott, T. 1996. Efficient translation of the RpoS sigma factor in Salmonella typhimurium requires Host Factor I, an RNA-binding protein encoded by the hfq gene. J Bacteriol 178:3763.

Brown, L. and Elliott, T. 1997. Mutations that increase expression of the rpos gene and decrease its dependence on $h f q$ function in Salmonella typhimurium. J Bacteriol 179:656.

Chatterjee, A., Cui, Y., Liu, Y., Dumenyo, C.K., and Chatterjee, A.K. 1995. Inactivation of rsmA leads to overproduction of extracellular pectinases, cellulases, and proteases in Erwinia carotovora subsp. carotovora in the absence of the starvation/cell density-sensing signal, 
N-(3-oxohexanoyl)-L-homoserine lactone. App/ Environ Microbiol 61:1959.

Collmer, A. and Keen, N.T. 1986. The role of pectic enzymes in plant pathogenesis. Annu Rev Phytopathol 24:383.

Cui, Y., Chatterjee, A., Liu Y., Dumenyo, C.K., and Chatterjee, A.K. 1995. Identification of a global repressor gene, rsmA, of Erwinia carotovora subsp. carotovora that controls extracellular enzymes, $\mathrm{N}$ (3-oxohexanoyl)-L-homoserine lactone, and pathogenicity in softrotting Erwinia spp. J Bacteriol 177:5108.

Cui, Y., Madi L., Mukherjee, A., Dumenyo, C.K., and Chatterjee, A.K. 1996. The RsmA- mutants of Erwinia carotovora subsp. carotovora strain Ecc71 overexpress hrpNEcc and elicit a hypersensitive reaction-like response in tobacco leaves. Mol Plant Microbe Interact 9:565.

Cui, Y., Mukherjee, A., Dumenyo, C.K., Liu, Y., and Chatterjee, A.K. 1999. rsmC of the soft-rotting bacterium Erwinia carotovora subsp. carotovora negatively controls extracellular enzyme and harpin(Ecc) production and virulence by modulating levels of regulatory RNA ( $r s m B)$ and RNA-binding protein (RsmA). J Bacteriol 181:6042.

Cui, Y., Chatterjee, A., and Chatterjee, A.K. 2001. Effects of the twocomponent system comprising GacA and GacS of Erwinia carotovora subsp. carotovora on the production of global regulatory rsmB RNA, extracellular enzymes, and harpinEcc. Mol Plant Microbe Interact 14:516.

Dubey, A.K., Baker, C.S., Suzuki, K., Jones, A.D., Pandit, P., Romeo, T., and Babitzke, P. 2003. CsrA regulates translation of the Escherichia coli carbon starvation gene, cstA, by blocking ribosome access to the cstA transcript. J Bacteriol 185:4450.

Fettes, P.S., Forsbach-Birk, V., Lynch, D., and Marre, R. 2001. Overexpresssion of a Legionella pneumophila homologue of the $E$. coli regulator CsrA affects cell size, flagellation, and pigmentation. Int J Med Microbiol 291:353.

Franze de Fernandez, M., Eoyang, L., and August, J. 1968. Factor fraction required for the synthesis of bacteriophage $\mathrm{Q} \beta$ RNA. Nature 219: 588

Geissmanns, S. and Touati, D. 2004. Hfq, a new chaperoning role: binding to messanger RNA determines access for small RNA regulator. EMBO J 23:396.

Gerdes, K., Gultyaev, A.P., Franch, T., Pedersen, K., and Mikkelsen, N.D. 1997. Antisense RNA-regulated programmed cell death. Annu Rev Genet 31:1.

Gudapaty, S., Suzuki K., Wang X., Babitzke P., and Romeo T. 2001. Regulatory interactions of Csr components: the RNA binding protein CsrA activates csrB transcription in Escherichia coli. J. Bacteriol 183: 6017.

Hajnsdorf, E. and Regnier, P. 2000. Host factor Hfq of Escherichia coli stimulates elongation of poly(A) tails by poly(A) polymerase I. Proc Natl Acad Sci USA 97:1501.

He, L. and Hannon, G. 2004. MicroRNAs: small RNAs with a big role in gene regulation. Nat Rev Genet. 5:522.

Heeb, S., Blumer, C., and Haas, D. 2002. Regulatory RNA as mediator in GacA/RsmA-dependent global control of exoproduct formation in Pseudomonas fluorescens CHAO. J Bacteriol 184:1046.

Hershberg, R., Altuvia, S., and Margalit, H. 2003. A survey of small RNAencoding genes in Escherichia coli. Nucleic Acids Res 31:1813.

Heurlier, K., Williams, F., Heeb, S., Dormond, C., Pessi, G., Singer, D., Camara, M., Williams, P., and Haas, D. 2004. Positive control of swarming, rhamnolipid synthesis, and lipase production by the posttranscriptional RsmA/RsmZ system in Pseudomonas aeruginosa PAO1. J Bacteriol. 186:2936.

Hyytiainen, H., Montesano, M., and Palva, E.T. 2001. Global regulators ExpA ( GacA) and KdgR modulate extracellular enzyme gene expression through the RsmA-rsmB system in Erwinia carotovora subsp. carotovora. Mol Plant Microbe Interact 14:931.

Isaacs, F.J., Dwyer, D.J., Ding, C., Pervouchine, D.D., Cantor, C.R., and Collins, J.J. 2004. Engineered riboregulators enable posttranscriptional control of gene expression. Nat Biotechnol 22: 841
Jackson, D.W., Suzuki, K., Oakford, L., Simecka, J.W., Hart, M.E., and Romeo, T. 2002. Biofilm formation and dispersal under the influence of the global regulator CsrA of Escherichia coli. J Bacteriol 184:290.

Kandror, O., DeLeon, A., and Goldberg, A. L. 2002. Trehalose synthesis is induced upon exposure of Escherichia coli to cold and is essential for viability at low temperatures. Proc Natl Acad Sci USA 99: 9727.

Kawano, M., Reynolds, A.A., Miranda-Rios, J., and Storz, G. 2005. Detection of 5'-and 3'-UTR-derived small RNAs and cis-encoded antisense RNAs in Escherichia coli. Nucl Acids Res 33:1040.

Klauck, E., Bohringer, J., and Hengge-Aronis, R. 1997. The LysR-like regulator LeuO in Escherichia coli is involved in the translational regulation of rpos by affecting the expression of the small regulatory DsrA-RNA. Molec Microbiol 25:559.

Lease, R.A., Cusick, M., and Belfort, M. 1998. Riboregulation in Escherichia coli: DsrA RNA acts by RNA: RNA interactions at multiple loci. Proc Natl Acad Sci USA 95:12456.

Lease, R.A. and Belfort, M. 2000a. A trans-acting RNA as a control switch in Escherichia coli: DsrA modulates function by forming alternative structures. Proc Natl Acad Sci USA 97:9919.

Lease, R.A. and Belfort, M. 2000b. Riboregulation by DsrA RNA: transactions for global economy. Mol Microbiol 38:667.

Lease, R.A., Smith, D., McDonough, K., and Belfort, M. 2004. The small noncoding DsrA RNA is an acid resistance regulator in Escherichia coli. J Bacteriol 186:6179.

Lease, R. A. and Woodson, S. A. 2004. Cycling of the Sm-like protein Hfa on the DsrA small regulatory RNA. J Molec Biol 344: 1211.

Lenz, D.H., Mok K.C., Lilley, B.N., Kulkarni, R.V., Wingreen, N.S., and Bassler, B.L. 2004. The Small RNA Chaperone Hfq and Multiple Small RNAs Control Quorum Sensing in Vibrio harveyi and Vibrio cholerae. Cell 118:69.

Liaw, S.J., Lai, H.C., Ho, S.W., Luh, K.T., and Wang, W.B. 2003. Role of RsmA in the regulation of swarming motility and virulence factor expression in Proteus mirabilis. J Med Microbiol 52:19.

Liu, M.Y., Yang, H., and Romeo, T. 1995. The product of the pleiotropic Escherichia coli gene csrA modulates glycogen biosynthesis via effects on mRNA stability. J Bacterio/ 177:2663.

Liu, M.Y., Gui, G., Wei, B., Preston, J.F., III, Oakford, L., Yuksel, U., Giedroc, D.P., and Romeo, T. 1997. The RNA molecule CsrB binds to the global regulatory protein CsrA and antagonizes its activity in Escherichia coli. J Biol Chem 272:17502.

Liu, Y., Cui, Y., Mukherjee, A., and Chatterjee, A.K. 1998. Characterization of a novel RNA regulator of Erwinia carotovora ssp. carotovora that controls production of extracellular enzymes and secondary metabolites. Mol Microbiol 29:219.

Liu, Y., Jiang, G., Cui Y., Mukherjee, A., Ma, W.L., and Chatterjee, A.K. 1999. kdgREcc negatively regulates genes for pectinases, cellulase, protease, HarpinEcc, and a global RNA regulator in Erwinia carotovora subsp. carotovora. J Bacteriol 181:2411.

Majdalani, N., Cunning, C., Sledjeski, D., Elliott, T., and Gottesman, S. 1998. DsrA RNA regulates translation of RpoS message by an antiantisense mechanism, independent of its action as an antisilencer of transcription. Proc Natl Acad Sci USA 95:12462.

Majdalani, N., Chen, S., Murrow, J., St. John, K., and Gottesman, S. 2001. Regulation of RpoS by a novel small RNA: the characterization of RprA. Mol Microbio/ 39:1382.

Majdalani, N., Hernandez, D., and Gottesman, S. 2002. Regulation and mode of action of the second small RNA activator of RpoS translation, RprA. Mol Microbiol 46:813.

Massé, E. and Gottesman, S. 2002. A small RNA regulates the expression of genes involved in iron metabolism in Escherichia coli. Proc Natl Acad Sci USA 99:4620.

Massé, E., Escorcia, F.E., and Gottesman, S. 2003. Coupled degradation of a small regulatory RNA and its mRNA targets in Escherichia coli. Genes Dev 17:2374.

Masuda, N. and Church, G.M. 2003. Regulatory network of acid resistance genes in Escherichia coli. Molec Microbiol 48:699. 
McManus, M.T. and Sharp, P.A. 2003. Gene Silencing in mammals by small interfering RNAs. Nature Reviews Genetics 3:737.

Mickulecky, P.J., Kaw, M.K., Brescia, C.C., Takach, J.C., Sledjeski, D.D., and Feig, A.L. 2004. Escherichia coli Hfq has distinct interaction surfaces for DsrA, rpoS and poly(A) RNAs. Nature Struct Molec Biol 11: 1206.

Mohanty, B.K., Maples, V.F., and Kushner, S.R. 2004. The Sm-like protein $\mathrm{Hfq}$ regulates polyadenylation dependent mRNA decay in Escherichia coli. Molec Microbiol 54:905.

Moll, I., Afonyushkin, T., Vytvytska, O., Kaberdin, V.R., and Blasi, U. 2003a. Coincident $\mathrm{Hfq}$ binding and RNase $\mathrm{E}$ cleavage sites on mRNA and small regulatory RNAs. RNA 9:1308.

Moll, I., Leitsch, D., Steinhauser, T., and Blasi, U. 2003b. RNA chaperone activity of the Sm-like Hfq protein. EMBO Rep 4:284.

Møller, T., Franch, T., Hojrup, P., Keene, D.R., Bachinger, H.P., Brennan, R., and Valentin-Hansen, P. 2002a. Hfq: a bacterial Sm-like protein that mediates RNA-RNA interaction. Mol Cell 9:23.

Møller, T., Franch, T., Udesen, C., Gerdes, K., and Valentin-Hansen, P. 2002b. Spot 42 RNA mediates discoordinate expression of the $E$. coli galactose operon. Genes Dev 16:1696.

Molofsky, A.B. and Swanson, M.S. 2003. Legionella pneumophila CsrA is a pivotal repressor of transmission traits and activator of replication. Mol Microbiol 50:445.

Morfeldt, E., Taylor, D., von Gabain, A., and Arvidson, S. 1995. Activation of alpha-toxin translation in Staphylococcus aureus by the transencoded antisense RNA, RNAll. EMBO J 14:4569.

Muffler, A., Fischer, D., and Hengge-Aronis, R., 1996. The RNA-binding protein HF-l, known as a host factor for phage QB RNA replication, is essential for rpoS translation in Escherichia coli. Genes Dev 10: 1143.

Murata, H., Chatterjee, A., Liu Y., and Chatterjee, A. K. 1994. Regulation of the production of extracellular pectinase, cellulase, and protease in the soft rot bacterium Erwinia carotovora subsp. carotovora: evidence that aepH of $E$. carotovora subsp. carotovora 71 activates gene expression in $E$. carotovora subsp. carotovora, $E$. carotovora subsp. atroseptica, and Escherichia coli. Appl Environ Microbiol 60:3150.

Pessi, G., Williams, F., Hindle, Z., Heurlier, K., Holden, M.T., Camara, M., Haas, D., and Williams, P. 2001. The global posttranscriptional regulator RsmA modulates production of virulence determinants and $\mathrm{N}$-acylhomoserine lactones in Pseudomonas aeruginosa. J Bacteriol 183:6676.

Reimmann, C., Valverde, C., Kay, E., and Haas, D. 2005. Posttranscriptional repression of GacS/GacA-controlled genes by the RNAbinding protein RsmE acting together with RsmA in the biocontrol strain Pseudomonas fluorescens CHAO. J Bacteriol 187: 276.

Repoila, F. and Gottesman, S. 2001. Signal transduction cascade for regulation of RpoS: Temperature regulation of DsrA. J Bacteriol. 183:4012.

Rivas, E., Klein, R.J., Jones, T.A., and Eddy, S.R. 2001. Computational identification of noncoding RNAs in $E$. coli by comparative genomics. Curr Biol 11:1369.

Romeo, T., Gong, M., Liu, M.Y., and Brun-Zinkernagel, A.M. 1993. Identification and molecular characterization of csrA, a pleiotropic gene from Escherichia coli that affects glycogen biosynthesis, gluconeogenesis, cell size, and surface properties. J Bacteriol 175: 4744.

Romeo, T. 1998. Global regulation by the small RNA-binding protein CsrA and the non-coding RNA molecule CsrB. Mol Microbiol 29: 1321.

Rowley, K.B., Clements, D.E., Mandel, M., Humphreys, T., and Patil, S. S. 1993. Multiple copies of a DNA sequence from Pseudomonas syringae pathovar phaseolicola abolish thermoregulation of phaseolotoxin production. Mol Microbiol 8:625.

Sabnis, N.A., Yang, H., and Romeo, T. 1995. Pleiotropic regulation of central carbohydrate metabolism in Escherichia coli via the gene csrA. J Biol Chem 270:29096.
Sauter, C., Basquin, J., and Suck, D. 2003. Sm-like proteins in eubacteria: the crystal structure of the Hfq protein from Escherichia coli. NuCl Acid Rese 31:4091.

Schultz, J.E. and Matin, A. 1991. Molecular and functional characterization of a carbon starvation gene of Escherichia coli. J Mol Biol 218:129.

Schumacher, M.A., Pearson, R.F., Møller, T., Valentin-Hansen, P., and Brennan, R.G. 2002. Structures of the pleiotropic translational regulator $\mathrm{Hfq}$ and an Hfq-RNA complex: a bacterial Sm-like protein. $E M B O$ J 21:3546.

Sledjeski, D. and Gottesman, S. 1995. A small RNA acts as an antisilencer of the H-NS-silenced rCSA gene of Escherichia coli. Proc Natl Acad Sci USA 92:2003.

Sledjeski, D.D., Gupta, A., and Gottesman, S. 1996. The small RNA, DsrA, is essential for the low temperature expression of RpoS during exponential growth in Escherichia coli. EMBO J 15:3993.

Sledjeski, D.D., Whitman, C., and Zhang, A. 2001. Hfq is necessary for regulation by the untranslated RNA DsrA. J Bacteriol. 183: 1997.

Stout, V. and Gottesman, S. 1991. Regulation of capsular polysaccharide synthesis in Escherichia coli K12. Mol Microbiol 5:1599.

Sukhodolets, M.V. and Garges, S. 2003. Interaction of Escherichia coli RNA polymerase with the ribosomal protein S1 and the Sm-like ATPase Hfq. Biochemistry 42:8022.

Sun, X., Zhulin, I., and Wartell, R.M. 2002. Predicted structure and phyletic distribution of the RNA-binding protein Hfq. Nucleic Acids Res 30:3662.

Suzuki, K., Wang, X., Weilbacher, T., Pernestig, A.K., Melefors, O., Georgellis, D., Babitzke, P., and Romeo, T. 2002. Regulatory circuitry of the CsrA/CsrB and BarA/UvrY systems of Escherichia coli. J Bacteriol 184:5130.

Takeda, S., Fujisawa, Y., Matsubara, M., Aiba, H., and Mizuno, T. 2001. A novel feature of the multistep phosphorelay in Escherichia coli: a revised model of the $\mathrm{Rcs} C \rightarrow$ YojN $\rightarrow$ RcsB signalling pathway implicated in capsular synthesis and swarming behavior. Mol Microbiol 40:440.

Valverde, C., Heeb, S., Keel, C., and Haas, D. 2003. RsmY, a small regulatory RNA, is required in concert with RsmZ for GacA-dependent expression of biocontrol traits in Pseudomonas fluorescens CHAO. Mol Microbiol 50:1361

Valverde, C., Lindell, M., Wagner, E.G., and Haas, D. 2004. A repeated GGA motif is critical for the activity and stability of the riboregulator RsmY of Pseudomonas fluorescens. J Biol Chem 279: 25066.

Vanderpool, C.K. and Gottesman, S. 2004. Involvement of a novel transcriptional activator and small RNA in post-transcriptional regulation of the glucose phosphoenolpyruvate phosphotransferase system. Molec Microbio/ 54:1076.

Vogel, J., Bartels, V., Tang H.H., Churakov, G., Slagter-Jager, J.G., Huttenhofer, A., and Wagner, E.G.H. 2003. RNomics in Escherichia coli detects new sRNA species and indicates parallel transcriptional output in bacteria. Nucl Acid Res 31:6435.

Wagner, E.G.H. and Brantl S. 1998. Kissing and RNA stability in antisense control of plasmid replication. Trends Biochem Sci 23:451.

Wassarman, K.M. and Storz, G. 2000. 6S RNA regulates E. coli RNA polymerase activity. Cell 101: 613.

Wassarman, K.M., Repoila, F., Rosenow, C., Storz, G., and Gottesman, S. 2001. Identification of novel small RNAs using comparative genomics and microarrays. Genes Dev 15:1637.

Waterman, S.R. and Small, P.L.C. 2003. Transcriptional expression of Escherichia coli Glutamate-dependent acid resistance genes gad $A$ and gadBC in an hns rpoS mutant. J Bacteriol 185:4644.

Wei, B.L., Brun-Zinkernagel, A.M., Simecka, J.W., Pruss, B.M., Babitzke, P., and Romeo, T. 2001. Positive regulation of motility and fIhDC expression by the RNA-binding protein CsrA of Escherichia coli. Mol Microbiol 40:245.

Weilbacher, T., Suzuki, K., Dubey, A.K., Wang, X., Gudapaty, S., Morozov, I., Baker, C.S., Georgellis, D., Babitzke, P., and Romeo, T. 2002. A 
novel sRNA component of the carbon storage regulatory system of Escherichia coli. Molec Microbiol 48:657.

White, D., Hart, M.E., and Romeo, T. 1996. Phylogenetic distribution of the global regulatory gene csrA among eubacteria. Gene 182: 221

Wilderman, P.J., Sowa, N.A., FitzGerald, D.J., FitzGerald, P.C., Gottesman, S., Ochsner, U.A., and Vasil, M.L. 2004. Identification of tandem duplicate regulatory small RNAs in Pseudomonas aeruginosa involved in iron homeostasis. Proc Natl Acad Sci USA 101:9792.

Worhunsky, D.J., Godek, K., Litsch, S., and Schlax, P.J. 2003. Interactions of non-coding RNA DsrA and RpoS mRNA with the 305 ribosomal subunit. J Biol Chem 278:15815.
Yang, H., Liu, M.Y., and Romeo, T. 1996. Coordinate genetic regulation of glycogen catabolism and biosynthesis in Escherichia coli via the CsrA gene product. J Bacteriol 178:1012.

Zhang, A., Altuvia, S., Tiwari, A., Argaman, L., Hengge-Aronis, R., and Storz, G. 1998. The oxyS regulatory RNA represses rpoS translation by binding $\mathrm{Hfq}(\mathrm{HF}-1)$ protein. EMBO J 17:6061.

Zhang, A., Wassarman, K.M., Ortega, J., Steven, A.C., and Storz, G. 2002. The Sm-like Hfa protein increases OxyS RNA interaction with target mRNAs. Mol Cell 9:11.

Zhang, A., Wassarman, K.M., Rosenow, C., Tjaden, B.C., Storz, G., and Gottesman, S. 2003. Global analysis of small RNA and mRNA targets of Hfq. Molec Microbiol 50:1111. 